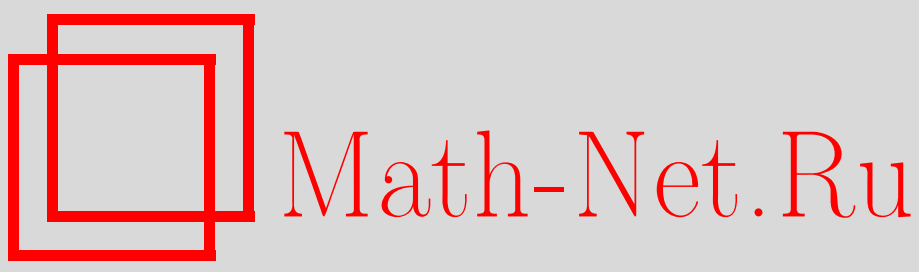

П. В. Морозов, Топология слоений Лиувилля случаев интегрируемости Стеклова и Соколова уравнений Кирхгофа, Матем. сб., 2004, том 195, номер 3, 69-114

DOI: https://doi.org/10.4213/sm809

Использование Общероссийского математического портала Math-Net.Ru подразумевает, что вы прочитали и согласны с пользовательским соглашением

http://www . mathnet.ru/rus/agreement

Параметры загрузки:

IP: 54.162 .85 .209

26 апреля 2023 г., 02:33:03 
УДК $515.1+521$

\author{
П.В. Морозов
}

\title{
Топология слоений Лиувилля случаев интегрируемости Стеклова и Соколова уравнений Кирхгофа
}

\footnotetext{
Статья посвящена исследованию топологии слоений Лиувилля случаев интегрируемости Стеклова и Соколова уравнений Кирхгофа. Вычисляются все изоэнергетические и круговые молекулы указанных гамильтоновых систем, дается классификация их невырожденных положений равновесия.

Библиография: 14 названий.
}

\section{§1. Интегрируемые гамильтоновы системы на симплектическом многообразии}

1.1. Понятие интегрируемой гамильтоновой системы. Рассмотрим гладкое $2 n$-мерное симплектическое многообразие $\left(M^{2 n}, \omega\right)$ с заданной на нем гладкой функцией $H$. Динамическую систему $v=\operatorname{sgrad} H$ назьвают гамильтоновой $\partial u$ намической системой с $n$ степенями свободы, а функцию $H$-ее гамильтонианом. (Векторное поле sgrad $H$ определяется тождеством $\omega(l, \operatorname{sgrad} H)=l(H)$, где $l$ - произвольный вектор касательного пространства, а $l(H)$ - производная функции $H$ вдоль $l$.) В локальных координатах $\left(x^{1}, \ldots, x^{2 n}\right)$ такую систему можно записать в виде системы обыкновенных дифференциальных уравнений:

$$
\dot{x}^{i}=\left\{x^{i}, H\right\}, \quad i=1, \ldots, 2 n .
$$

Здесь $\{\cdot, \cdot\}$ - скобка Пуассона на многообразии $M^{2 n}$, определяемая симлектической структурой $\omega$ посредством тождества:

$$
\{f, g\}=\omega(\operatorname{sgrad} f, \operatorname{sgrad} g) .
$$

ОПРЕДЕЛЕНИЕ 1. Гамильтонова система $v$ называется вполне интегрируемой nо Лиувиллю, если существует набор гладких функций $f_{1}, \ldots, f_{n}$ таких, что

1) $f_{1}, \ldots, f_{n}$ - первые интегралы $v$ (что равносильно условию $\left\{f_{i}, H\right\}=0$, выполненному при любом $i$ на всем $M$ ),

2) они функционально независимы, т.е. почти всюду на $M$ их градиенты линейно независимы,

3) $\left\{f_{i}, f_{j}\right\}=0$ при любых $i$ и $j$,

4) векторные поля $\operatorname{sgrad} f_{i}$ полны.

Часто для краткости вполне интегрируемые по Лиувиллю системы называют просто интегрируемыми.

(C) П. в. Морозов 2004 
ОПРЕДЕЛЕНИЕ 2. Слоением Лиувилля, отвечающим вполне интегрируемой системе, называется разбиение многообразия $M^{2 n}$ на связные компоненты совместных поверхностей уровня интегралов $f_{1}, \ldots, f_{n}$.

1.2. Теорема Лиувилля. Топология вполне интегрируемой гамильтоновой системы в окрестности совместной регулярной поверхности уровня ее первых интегралов полностью описывается теоремой Лиувилля. Обозначим поверхность уровня через $T_{\xi}$ :

$$
T_{\xi}=\left\{x \in M \mid f_{i}(x)=\xi_{i}, i=1, \ldots, n\right\}
$$

Регулярность означает, что дифференциалы $d f_{i}$ линейно независимы на $T_{\xi}$.

ТЕорема 1 (теорема Лиувилля). Пусть на $M^{2 n}$ задана вполне интегрируемая по Лиувиллю гамильтонова система $v=\operatorname{sgrad} H u T_{\xi}-$ регулярная совместная поверхность уровня интегралов $f_{1}, \ldots, f_{n}$. Тогда

1) $T_{\xi}$ - гладкое лагранжсево подмногообразие, инвариантное относительно потоков $v=\operatorname{sgrad} H u \operatorname{sgrad} f_{1}, \ldots, \operatorname{sgrad} f_{n}$;

2) если многообразие $T_{\xi}$ компактно, то кажсдая компонента связности $T_{\xi}$ диффеоморфна п-мерному тору $T^{n}$. Такие торь называются торами Лиувилля;

3) слоение Лиувилля в некоторой окрестности $U$ тора Лиувилля $T_{\xi}$ тривиально, т.е. диффеоморфно прямому произведению тора $T^{n}$ на диск $D^{n}$;

4) в окрестности $U=T^{n} \times D^{n}$ существует система координат $s_{1}, \ldots, s_{n}$, $\varphi_{1}, \ldots, \varphi_{n}$, называемых переменные действие-угол, со свойствами:

а) $s_{1}, \ldots, s_{n}-$ координаты на диске $D^{n}, \varphi_{1}, \ldots, \varphi_{n}-$ стандартные угловые координаты на торе $T^{n}, \varphi \in \mathbb{R} / 2 \pi \mathbb{Z}$;

б) $\omega=\sum d \varphi_{i} \wedge d s_{i}$

в) переменные действия $s_{i}$ являются функииями интегралов $f_{1}, \ldots, f_{n}$;

г) в переменных действие-угол гамильтонов поток выпрямляется на каждом торе Лиувилля из окрестности $U$ и гамильтоновы уравнения принимают вид

$$
\dot{s}_{i}=0, \quad \dot{\varphi}_{i}=q_{i}\left(s_{1}, \ldots, s_{n}\right), \quad i=1, \ldots, n .
$$

Это означает, что на каждом торе поток $v$ задает условно периодическое движение, а траектории являются прямолинейными обмотками тора (рациональным.ми или иррачиональными).

Доказательство теоремы можно найти в [1].

\section{3. Лиувиллева эквивалентность интегрируемых гамильтоновых} систем. В теории топологической классификации интегрируемых гамильтоновых систем традиционно рассматриваются несколько типов их изоморфизмов. Настоящая работа посвящена классификации систем Стеклова [2] и Соколова [3] из механики твердого тела с точностью до лиувиллевой эквивалентности. 
ОПРЕДЕЛЕНИЕ 3. Две интегрируемые гамильтоновы системы $(M, v)$ и $\left(M^{\prime}, v^{\prime}\right)$ называются лиувиллево әквивалентными, если существует гомеоморфизм $\Phi$ : $M \rightarrow M^{\prime}$, переводящий лиувиллево слоение первой системы в лиувиллево слоение второй системы.

Это отношение эквивалентности можно несколько ослабить. В результате возникает понятие грубой лиувиллевой эквивалентности.

ОПРЕДЕЛЕНИЕ 4. Две интегрируемые гамильтоновы системы $(M, v)$ и $\left(M^{\prime}, v^{\prime}\right)$ называются грубо лиувиллево әквивалентнылии, если сушествует гомеоморфизм между базами соответствующих слоений Лиувилля, который локально (т.е. в окрестности каждой точки) поднимается до послойного гомеоморфизма слоений Лиувилля.

\section{§ 2. Инвариаты Фоменко-Цишанга интегрируемых гамильтоновых систем с двумя степенями свободы}

Будем далее рассматривать интегрируемые гамильтоновы системы с двумя степенями свободы, т.е. случай $n=2$. Тогда интегрируемость системы на $M^{4}$ гарантируется сушествованием лишь одного функционально независимого с гамильтонианом $H$ интеграла $f$. В этом параграфе мы опишем основные этапы построения известного инварианта Фоменко-Цишанга (или меченой молекулы) [1], который описывает глобальную структуру лиувиллева слоения на неособых трехмерных изоэнергетических подмногообразиях фазового пространства $M^{4}$ интегрируемой гамильтоновой системы с двумя степенями свободы.

2.1. Изоэнергетические поверхности. Изоэнергетическими поверхностямм называются трехмерные поверхности вида $Q_{h}^{3}=\left\{x \in M^{4} \mid H(x)=h\right\}$. Сразу ограничимся рассмотрением лишь тех $h$, при которых, во-первых, $Q_{h}^{3}$ компактна и, во-вторых, $d H \neq 0$ всюду на $Q_{h}^{3}$. Тем самым мы гарантируем, что $Q_{h}^{3}$ является гладким компактным подмногообразием в $M^{4}$, а векторное поле $v=$ sgrad $H$ нигде не обрашается в нуль.

Наряду с лиувиллевой эквивалентностью на всем симлектическом многообразии мы будем говорить о лиувиллевой эквивалентности на избранных изоэнергетических поверхностях. Для того чтобы строго ввести это понятие, достаточно в определениях 3 и 4 заменить $M^{4}$ на $Q^{3}$.

ОПРЕДЕЛЕНИЕ 5 . Точку $x \in Q^{3}$ будем называть критической, если векторы $\operatorname{sgrad} H$ и $\operatorname{sgrad} f$ в ней линейно зависимы.

Заметим, что сингулярные совместные поверхности уровня интегралов $T_{\xi}$ в $Q^{3}$ - это в точности те поверхности, на которые попали критические точки, и теорема Лиувилля к ним не применима.

Сделаем еше одно предположение о изоэнергетической поверхности, касаюшееся свойств критических точек. Легко показать, что критические точки на $Q^{3}$ не могут быть изолированными. Поэтому предполагать, что дополнительный интеграл $f$ является функцией Морса, бессмысленно. Однако в случае динамических систем существует естественный аналог этого понятия. 
ОПРЕДЕЛЕНИЕ 6. Дополнительный интеграл $f$ назьвается интегралом Бот$m a$ на данной изоэнергетической поверхности $Q^{3}$, если все его критические точки организованы в невырожденные критические подмногообразия.

Это означает, что множество критических точек является несвязным объединением некоторых гладких подмногообразий, причем каждое из них невырождено в следующем смысле. Второй дифференциал $d^{2} f$ невырожден на подпространстве, трансверсальном к подмногообразию (в каждой точке). Другими словами, ограничение функции $f$ на трансверсаль к подмногообразию является функцией Морса.

В реальных интегрируемых задачах физики и механики типична ситуация, когда найденный дополнительньй интеграл $f$ является боттовским для всех $h$, кроме некоторого конечного набора значений. Случаев же, когда неботтовские значения $h$ отсутствуют полностью, известно крайне мало.

2.2. Структура критических множеств на изоэнергетической поверхности. Условие боттовости налагает существенное ограничение на структуру множества критических точек в $Q^{3}$. Действительно, каждая компонента его связности должна быть гладким компактньм подмногообразием размерности 1 или 2. Из условия $v=\operatorname{sgrad} H \neq 0$ следует, что на этих подмногообразиях существует гладкое векторное поле, отличное от нуля в каждой точке. Следовательно, это или окружность, или двумерный тор, или бутылка Клейна. В известных примерах математической физики критические множества двух последних типов встречаются крайне редко. Топология таких особенностей подробно изучена в [1]. Мы же далее в этом параграфе будем предполагать, что все критические подмногообразия являются окружностями.

2.3. Окрестности сингулярных слоев лиувиллева слоения на изоэнергетической поверхности. Изоэнергетическая поверхность $Q^{3}$ представляет собой однопараметрическое семейство совместных поверхностей уровня $T_{\xi}$ интегралов системы $H$ и $f$, параметризованное значением второго интеграла $f$. Если стянуть каждую компоненту связности поверхностей $T_{\xi}$ в точку, то мы получим некоторьй одномерньй граф - базу слоения Лиувилля (см. рис. 1). Прообразом каждого ребра такого графа является многообразие, диффеоморфное $T^{2} \times(0,1)$. Вершинам графа соответствуют сингулярные слои. Типичной является ситуация, когда при переходе через критический уровень число компонент связности $T_{\xi}$ меняется.

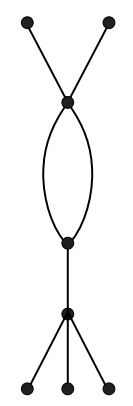

Рис. 1 
Будем рассматривать трехмерную окрестность особого слоя в $Q^{3}$. Оказывается, что в боттовском случае с точностью до лиувиллевой эквивалентности существует лишь конечное число возможных перестроек (бифуркаций), если фиксировать количество критических окружностей на сингулярном слое.

ОПРЕДЕЛЕНИЕ 7. Класс лиувиллевой эквивалентности окрестности особого слоя слоения Лиувилля называется 3-атомом.

По сути, 3-атом - это трехмерное многообразие со структурой лиувиллева слоения, содержашего ровно один сингулярный слой. Край такого многообразия состоит из некоторого числа несвязных торов Лиувилля. Количество критических окружностей на сингулярном слое называется сложностью 3-атома. В [1] изложен алгоритм, позволяющий явно перечислить все атомы заданной сложности. 3 -атомы принято обозначать заглавными латинскими буквами с нату ральньми индексами и звездочками. Четыре наиболее простых и типичных 3 -атома $\left(A, A^{*}, B\right.$ и $C_{2}$ ) изображены на рис. 2.

Если теперь в вершинах графа на рис. 1 поставить подходящие 3-атомы, то мы получим так называемую грубую молекулу. Грубая молекула несет информацию о базе слоения Лиувилля, а также позволяет локально восстановить его структуру вблизи как регулярных, так и сингулярных слоев. Справедлива

Tеорема 2 (А.Т. Фоменко [1]). Две интегрируемые гамильтоновы системиц $\left(v, Q^{3}\right)$ и $\left(v^{\prime}, Q^{\prime 3}\right)$ грубо лиувиллево әквивалентньц в том и только том случае, когда их грубые молекулы совпадают.

Полное и последовательное доказательство фактов, изложенных в этом пункте, можно найти в [1]. Здесь мы сформулируем основную теорему, на которую опираются эти доказательства.

Теорема 3 (А.Т. Фоменко [1]). 1) Трехмерная окрестность сингулярного слоя лиувиллева слоения является расслоением Зейферта, особъе слои которого (если они существуют) имеют тип $(2,1)$.

2) Если особых слоев у этого расслоения нет, то окрестность представима в виде прямого произведения $P^{2} \times S^{1}$, әде $P^{2}$ - ориентируемая поверхность с краем из несвязных окружностей.

3) Структура расслоения Зейферта согласована со структурой лиувиллева слоения в том смысле, что всякий слой расслоения Зейферта (окружность) иеликом лежит на некотором слое слоения Лиувилля.

2.4. Склейка изоэнергетической поверхности из 3-атомов. 3-атомы описывают локальную структуру слоения Лиувилля в окрестности особого слоя. Для того чтобы восстановить структуру слоения глобально на всем $Q^{3}$, нужно знать гомеоморфизмы, по которым склеены граничные торы 3-атомов. Если на каждом из пары склеиваемых торов выбрать базисные циклы, то склеивающий гомеоморфизм задается целочисленной матрищей $2 \times 2$ с определителем \pm 1 . Но базисы можно выбирать многими разными способами.

Оказывается, однако, что всякий 3-атом определяет на своих граничных торах некоторый цикл, называемый однозначно определенным циклом данной бифуркации. В случае атома $A$ это цикл, который стягивается в точку при приближении 

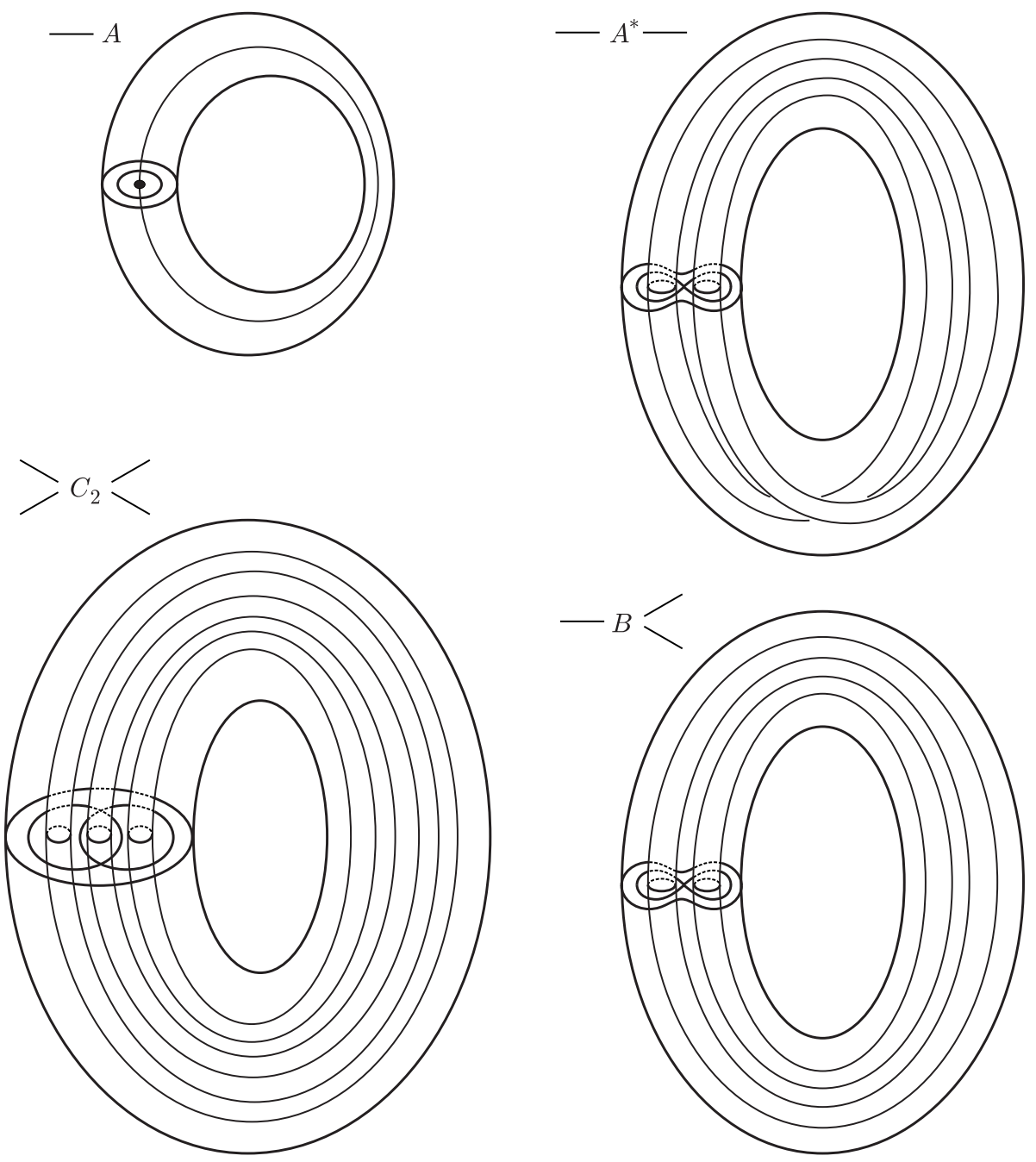

Рис. 2

к критической окружности. А в случае всех остальных (седловых) атомов $\left(A^{*}, B\right.$, $C_{2}, \ldots$ ) это цикл, изотопный слоям расслоения Зейферта.

Выделить подобным образом какой-то один цикл из множества кандидатов на вторую позицию в базисе не удается. Поэтому ограничиваются выбором сразу множества циклов, обладающих некоторыми общими свойствами. Вместе с первьгм циклом они составляют класс так называемых допустимых систем координат для данного атома. Точное определение см. в [1]. При этом группа замен внутри класса допустимых систем координат имеет уже очень простую структуру. Инвариантами действия этой группы на множестве матриц склеек являются числовые метки $r$, $\varepsilon$ и $n$. Они могут быть легко вычислены по этим матрицам.

Пара меток $r$ и в возникает на каждом ребре молекулы. А метка $n$ может возникнуть на седловом атоме или сразу на групше седловых атомов, называемых семьей. 
Числовые метки имеют естественный топологический смысл.

Так, метка $r \in\{\mathbb{Q} \cap[0,1), \infty\}$ на ребре определяет беззнаковый индекс пересечения однозначно определенных циклов бифуркаций, которые соединены этим ребром. Метка $\varepsilon \in\{1,-1\}$ говорит о сохранении или изменении ориентации критических окружностей двух бифуркаций (когда такое сравнение корректно определено). А метка $n \in \mathbb{Z}$ есть число Эйлера некоторого расслоения Зейферта, которое образуется при склейке атомов.

Наконец, дадим определение инварианта Фоменко-Цишанга и сформулируем основную теорему этого пункта.

ОПРЕДЕЛЕниЕ 8. Грубая молекула $W$, снабженная метками $r_{i}, \varepsilon_{i}$ и $n_{k}$, называется инвариантом Фоменко-Цишанга или просто меченой молекулой.

Теорема 4 (А.Т. Фоменко, Х. Цишанг [1]). Две интегрируемве гамильтоновы системь $\left(v, Q^{3}\right)$ и $\left(v^{\prime}, Q^{\prime 3}\right)$ лиувиллево әквивалентны в том и только том случае, когда их меченье молекуль совпадают.

\section{§3. Лиувиллева классификация интегрируемого случая Стеклова}

3.1. Фазовое пространство. Определим пару $\left(M^{4}, \omega\right)$, возникающую в динамике твердого тела.

Рассмотрим алгебру Ли $е(3)$ группы Ли $E(3)$ движений трехмерного евклидова пространства. На линейном пространстве $e(3)^{*}$ определена скобка Ли-Пуассона двух произвольных гладких функций $f$ и $g$ :

$$
\{f, g\}(x)=x\left(\left[d_{x} f, d_{x} g\right]\right),
$$

где $x \in e(3)^{*}, \mathrm{a}[\cdot, \cdot \cdot]$ - коммутатор в алгебре Ли $e(3)$.

В естественных координатах $\left(s_{1}, s_{2}, s_{3}, r_{1}, r_{2}, r_{3}\right), s=\left(s_{1}, s_{2}, s_{3}\right), r=\left(r_{1}, r_{2}, r_{3}\right)$, на пространстве $e(3)^{*}$ эта скобка записьвается следующим образом:

$$
\left\{s_{i}, s_{j}\right\}=\varepsilon_{i j k} s_{k}, \quad\left\{r_{i}, s_{j}\right\}=\varepsilon_{i j k} r_{k}, \quad\left\{r_{i}, r_{j}\right\}=0,
$$

где

$$
1 \leqslant i, j, k \leqslant 3, \quad \varepsilon_{i j k}=\frac{1}{2}(i-j)(j-k)(k-i) .
$$

Пусть на $e(3)^{*}$ задана функция Гамильтона $H(s, r)$. Рассмотрим систему уравнений:

$$
\dot{s}_{i}=\left\{s_{i}, H\right\}, \quad \dot{r}_{i}=\left\{r_{i}, H\right\} .
$$

Функции $f_{1}=r_{1}^{2}+r_{2}^{2}+r_{3}^{2}$ и $f_{2}=s_{1} r_{1}+s_{2} r_{2}+s_{3} r_{3}$ лежат в ядре скобки Ли-Пуассона и поэтому являются первыми интегралами уравнений (1). Несложно показать, что на совместных четырехмерных поверхностях уровня функций $f_{1}$ и $f_{2}$ :

$$
M_{g}^{4}=\left\{f_{1}=r_{1}^{2}+r_{2}^{2}+r_{3}^{2}=1, f_{2}=s_{1} r_{1}+s_{2} r_{2}+s_{3} r_{3}=g\right\},
$$

ограничение системы (1) представляет собой гамильтонову систему с двумя степенями свободы. Поверхности $M_{g}^{4}$ являются неособыми гладкими подмногообразиями в $e(3)^{*}$, диффеоморфными $T S^{2}$. Сиплектическая структура задается ограничением скобки Ли-Пуассона из объемлюшего пространства $e(3)^{*}$. Система будет 
интегрируемой, если существует функционально независимая с $H$ гладкая функция $F(s, r)$ такая, что $\{H, F\}=0$.

Итак, каждая функция $H(s, r)$, заданная на всем $e(3)^{*}$, порождает однопараметрическое семейство гамильтоновых систем с двумя степенями свободы. Параметр $g$ имеет физический смысл постоянной площадей.

3.2. Случаи интегрируемости Эйлера, Клебша и Стеклова. Фундаментальную роль в механике твердого тела играет случай интегрируемости Эйлеpa [4], которьй соответствует свободному движению твердого тела, закрепленного в центре масс. Укажем конкретный вид гамильтониана $H$ и дополнительного интеграла $F$ случая Эйлера:

$$
H=\frac{s_{1}^{2}}{2 A_{1}}+\frac{s_{2}^{2}}{2 A_{2}}+\frac{s_{3}^{2}}{2 A_{3}}, \quad F=s_{1}^{2}+s_{2}^{2}+s_{3}^{2} .
$$

Здесь вешественные параметры $A_{i}$ имеют смысл главных моментов инерции твердого тела.

Хорошо известны также случаи интегрируемости Клебша [5] и Стеклова [2], которые соответствуют задаче о движении твердого тела в жидкости:

случай Клебша -

$$
\begin{aligned}
H & =\frac{s_{1}^{2}}{2 A_{1}}+\frac{s_{2}^{2}}{2 A_{2}}+\frac{s_{3}^{2}}{2 A_{3}}+\frac{\varepsilon}{2}\left(A_{1} r_{1}^{2}+A_{2} r_{2}^{2}+A_{3} r_{3}^{2}\right), \\
F & =\left(s_{1}^{2}+s_{2}^{2}+s_{3}^{2}\right)-\varepsilon\left(A_{2} A_{3} r_{1}^{2}+A_{1} A_{3} r_{2}^{2}+A_{1} A_{2} r_{3}^{2}\right) ;
\end{aligned}
$$

случай Стеклова -

$$
\begin{aligned}
H= & \frac{s_{1}^{2}}{2 A_{1}}+\frac{s_{2}^{2}}{2 A_{2}}+\frac{s_{3}^{2}}{2 A_{3}}+\varepsilon\left(A_{1} r_{1} s_{1}+A_{2} r_{2} s_{2}+A_{3} r_{3} s_{3}\right) \\
& +\frac{\varepsilon^{2}}{2}\left(A_{1}\left(A_{2}^{2}+A_{3}^{2}\right) r_{1}^{2}+A_{2}\left(A_{3}^{2}+A_{1}^{2}\right) r_{2}^{2}+A_{3}\left(A_{1}^{2}+A_{2}^{2}\right) r_{3}^{2}\right), \\
F= & \left(s_{1}^{2}+s_{2}^{2}+s_{3}^{2}\right)-2 \varepsilon\left(A_{2} A_{3} s_{1} r_{1}+A_{3} A_{1} s_{2} r_{2}+A_{1} A_{2} s_{3} r_{3}\right) \\
& +\varepsilon^{2}\left(A_{1}^{2}\left(A_{2}-A_{3}\right)^{2} r_{1}^{2}+A_{2}^{2}\left(A_{3}-A_{1}\right)^{2} r_{2}^{2}+A_{3}^{2}\left(A_{1}-A_{2}\right)^{2} r_{3}^{2}\right) .
\end{aligned}
$$

Здесь $\varepsilon \in \mathbb{R}$ - некоторый действительный параметр.

Как видно, эти два случая интегрируемости можно интерпретировать как однопараметрические возмущения случая Эйлера в классе интегрируемых систем.

Лиувиллева классификация случая Эйлера изложена в [1]. В работе [6] при помощи метода круговых молекул, предложенного А. В. Болсиновым, П. Рихтером, А.Т. Фоменко в [7], были вычислены все меченые молекулы случая Клебша. В настояшем параграфе будут вычислены инварианты Фоменко-Цишанга для случая Стеклова. В следуюшем параграфе вычисляются меченые молекулы для случая Соколова [3]. Здесь также основным аппаратом является метод круговых молекул [7]. 
В заключение дадим ряд ссылок на другие работы по этой тематике. Грубая лиувиллева классификация случаев интегрируемости Эйлера-Жуковского, Лагранжа, Ковалевской, Горячева-Чаплыгина, Сретенского, Клебша, Стеклова и четырехмерного твердого тела была получена А. А. Ошемковьм [8], [9]. Грубые молекулы случая Соколова вычислены П.Е. Рябовым [10]. Тонкая лиувиллева классификация волчков Эйлера и Лагранжа подробно изложена в [1]. В работе [7] авторами вычислены все меченые молекулы случая Ковалевской при помощи предложенного ими метода круговых молекул. Метки молекул случая частичной интегрируемости Горячева-Чапльпина были получены Орел [11]. Также следует отметить работу Топалова [12], в которой автору при помощи введенных им $n$-соотношений удалось вычислить $n$-метки для многих случаев интегрируемости механики твердого тела.

3.3. Грубая лиувиллева классификация систем случая Стеклова. Грубые молекулы для интегрируемого случая Стеклова вычислены А. А. Ошемковым в работе [9]. В этом пункте мы вкратце приводим его результаты и вводим понятия и обозначения, которые понадобятся нам для вычисления тонких лиувиллевых инвариантов.

Рассмотрим коммутирующую пару функций на $e(3)^{*}$ :

$$
\begin{aligned}
H_{0} & =a_{1} s_{1}^{2}+a_{2} s_{2}^{2}+a_{3} s_{3}^{2}+2\left(a_{1}^{2} s_{1} r_{1}+a_{2}^{2} s_{2} r_{2}+a_{3}^{2} s_{3} r_{3}\right)+a_{1}^{3} r_{1}^{2}+a_{2}^{3} r_{2}^{2}+a_{3}^{3} r_{3}^{2} \\
F_{0} & =s_{1}^{2}+s_{2}^{2}+s_{3}^{2}-2\left(a_{1} s_{1} r_{1}+a_{2} s_{2} r_{2}+a_{3} s_{3} r_{3}\right)-3\left(a_{1}^{2} r_{1}^{2}+a_{2}^{2} r_{2}^{2}+a_{3}^{2} r_{3}^{2}\right)
\end{aligned}
$$

где $a_{1}+a_{2}+a_{3}=0$. Как впервые заметил А. А. Ошемков, любой гамильтониан $H$ случая Стеклова представим в виде

$$
H=\alpha H_{0}+\beta F_{0}+\gamma f_{1}+\delta f_{2},
$$

где

$$
\begin{aligned}
a_{1} & =\frac{\varepsilon}{3}\left(2 A_{2} A_{3}-A_{3} A_{1}-A_{1} A_{2}\right), \\
a_{2} & =\frac{\varepsilon}{3}\left(2 A_{3} A_{1}-A_{1} A_{2}-A_{2} A_{3}\right), \\
a_{3} & =\frac{\varepsilon}{3}\left(2 A_{1} A_{2}-A_{2} A_{3}-A_{3} A_{1}\right), \\
\alpha & =\frac{1}{2 \varepsilon A_{1} A_{2} A_{3}}, \quad \beta=\frac{1}{6}\left(\frac{1}{A_{1}}+\frac{1}{A_{2}}+\frac{1}{A_{3}}\right), \\
\gamma & =\varepsilon^{2}\left(\frac{2\left(A_{1} A_{2}+A_{2} A_{3}+A_{3} A_{1}\right)^{3}}{27 A_{1} A_{2} A_{3}}-A_{1} A_{2} A_{3}\right), \\
\delta & =\frac{\varepsilon}{9}\left(5\left(A_{1}+A_{2}+A_{3}\right)-2\left(\frac{A_{2} A_{3}}{A_{1}}+\frac{A_{3} A_{1}}{A_{2}}+\frac{A_{1} A_{2}}{A_{3}}\right)\right) .
\end{aligned}
$$

Далее будем рассматривать интегрируемую систему с гамильтонианом $H_{0}$ и дополнительньм интегралом $F_{0}$. Тем самым мы фактически понижаем число параметров с четырех до двух. Перенумерацией переменных добьемся, чтобы

$$
a_{1}<0 \leqslant a_{2}<a_{3} .
$$


Рассмотрим отображение момента $\mathfrak{F}$, которое определяется следующим обра30м:

$$
\mathfrak{F}: M_{g}^{4} \rightarrow \mathbb{R}^{2}(f, h), \quad \mathfrak{F}: x \rightarrow\left(F_{0}(x), H_{0}(x)\right) .
$$

Образ критических точек при отображении момента назьвается бифуркационной диаграммой. В случае Стеклова при любом значении $g$ бифуркационная диаграмма состоит из кривой, заданной параметрически:

$$
f=-8 \mu g-12 \mu^{2}, \quad h=-4 \mu^{2} g-8 \mu^{3}, \quad-\left(g+a_{3}\right) \leqslant 2 \mu \leqslant-\left(g+a_{1}\right),
$$

и трех лучей, лежащих на прямых $h=a_{i} f+4 a_{i}^{3}+4 g a_{i}^{2}(i=1,2,3)$. Лучи задаются так: $\left\{h=a_{i} f+4 a_{i}^{3}+4 g a_{i}^{2}, f \geqslant T-12 a_{i}^{2}-8 g a_{i}\right\}$, где $T$ - некоторое неотрицательное число.

Кривая (2), заданная параметрически с параметром $\mu$, имеет вид, изображенньй на рис. 3 :
(a) $g<-3 a_{3}$,
(b) $-3 a_{3}<g<-3 a_{2}$,
(c) $-3 a_{2}<g<-3 a_{1}$,
(d) $g>-3 a_{1}$.

(a) $\uparrow h$

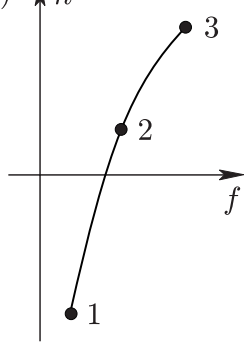

(b)

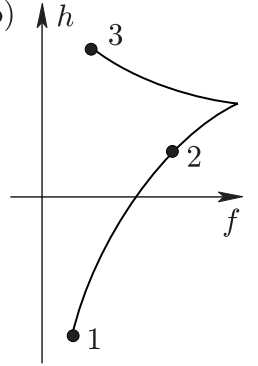

(c) $1 h$

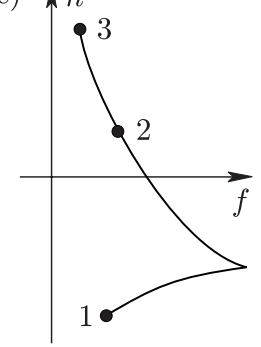

(d) $\uparrow h$

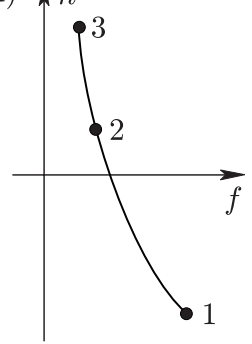

Рис. 3

Для точки кривой, отмеченной цифрой $i$, значение параметра равно $-\left(g+a_{i}\right) / 2$. Это точка пересечения кривой и прямой $h=a_{i} f+4 a_{i}^{3}+4 g a_{i}^{2}$. Для точки возврата кривой мы имеем $\mu=g / 3$ при $-3 a_{3}<g<-3 a_{1}$. В точке касания луча с кривой значение параметра на кривой равно $a_{i}$.

В результате для различных значений $g$ получаем в общей сложности 10 бифуркационных диаграмм. Они изображены на рис. 4. Соответствующие значения $g$ таковы:
a) $g<-3 a_{3}$,
f) $a_{2}-a_{3}<g<a_{3}-a_{2}$,
b) $-3 a_{3}<g<a_{1}-a_{3}$,
g) $a_{3}-a_{2}<g<a_{2}-a_{1}$,
c) $a_{1}-a_{3}<g<a_{1}-a_{2}$,
h) $a_{2}-a_{1}<g<a_{3}-a_{1}$,
d) $a_{1}-a_{2}<g<-3 a_{2}$,
i) $a_{3}-a_{1}<g<-3 a_{1}$,
e) $-3 a_{2}<g<a_{2}-a_{3}$,
j) $g>-3 a_{1}$.

На рис. 4 гладкие дуги бифуркационной диаграммы обозначены мальми греческими буквами с индексами. Окрестности их прообразов в $Q^{3}$ представляют собой боттовские перестройки торов Лиувилля, описываемые 3 -атомами. Укажем их типы: 
a)

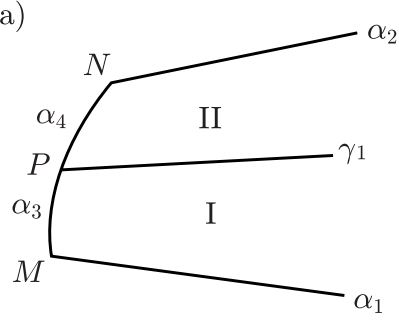

c)

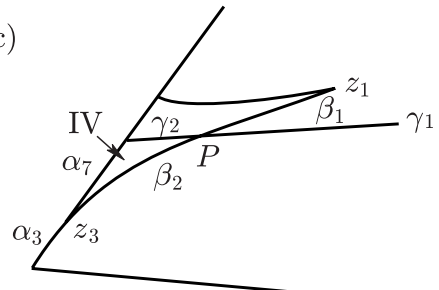

e)

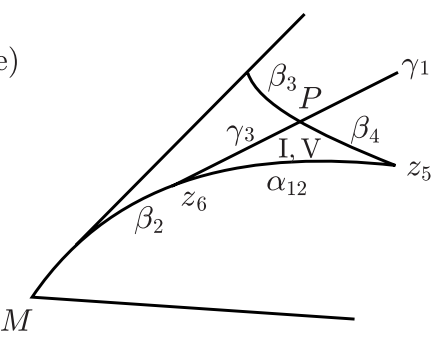

g)

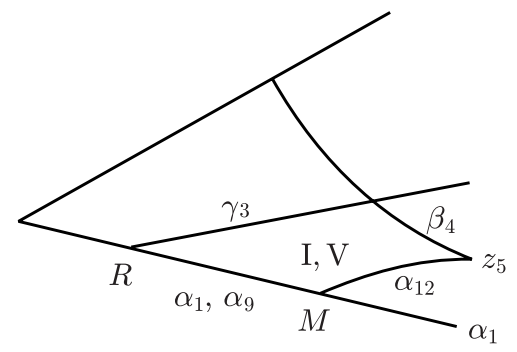

i)

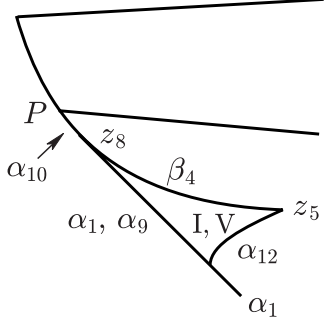

b)

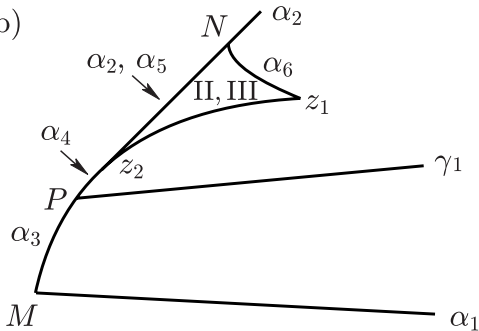

d)

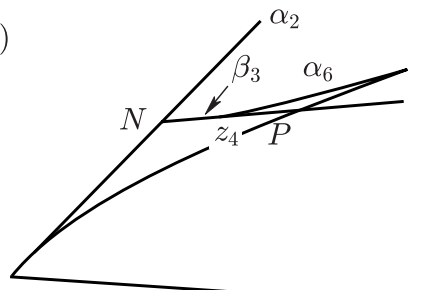

f)

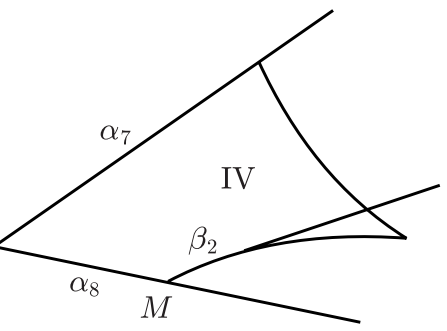

h)

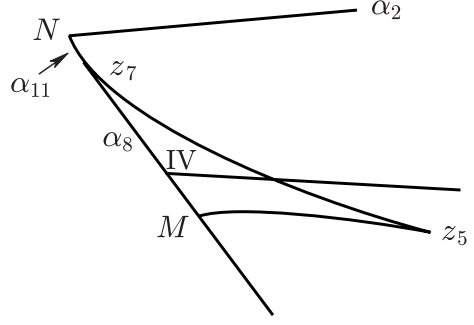

j)

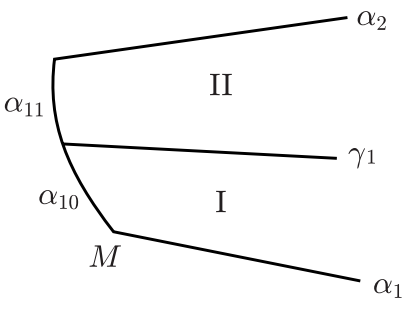

Рис. 4 
$2 A: \quad \alpha_{1}, \alpha_{2}, \alpha_{3}, \alpha_{4}, \alpha_{5}, \alpha_{6}, \alpha_{9}, \alpha_{10}, \alpha_{11}, \alpha_{12}$,

$4 A: \quad \alpha_{7}, \alpha_{8}$,

$2 B: \beta_{1}, \beta_{2}, \beta_{3}, \beta_{4}$,

$C_{2}: \gamma_{1}$,

$2 C_{2}: \gamma_{2}, \gamma_{3}$.

Регулярные точки отображения момента на $\mathbb{R}^{2}(f, h)$ являются образами некоторого количества несвязных торов Лиувилля. Из результатов работы Ошемкова [9] вытекает, что эти торы естественным образом разбиваются на семейства, которые мы будем обозначать римскими цифрами I-V (см. рис. 4). Число торов Лиувилля для каждой области регулярности также вычислено в [9]. Эта информация представлена в таблице 1.

ТАБЛИЦА 1

\begin{tabular}{|c|c|}
\hline Семейство & $\begin{array}{c}\text { Число торов } \\
\text { Лиувилля }\end{array}$ \\
\hline I & 2 \\
\hline II & 2 \\
\hline III & 2 \\
\hline IV & 4 \\
\hline V & 2 \\
\hline
\end{tabular}

В каждое семейство мы отнесли торы, которые испытьвают одинаковые бифуркации на границах области регулярности. Появление пар торов-близнецов не должно нас удивлять. Дело в том, что фазовое пространство обладает очевидной симметрией

$$
\Phi:(s, r) \rightarrow(-s,-r)
$$

такой, что $\Phi:\left(f_{1}, f_{2}, H_{0}, F_{0}\right) \rightarrow\left(f_{1}, f_{2}, H_{0}, F_{0}\right)$.

Особо отметим, что регулярные торы Лиувилля могут лежать и в прообразах кривых бифуркационной диаграммы, как это происходит, например, с дугой $\beta_{1}$. Дело в том, что кривая $\beta_{1}$ является бифуркационной только для семейства III, а для семейства II торов эта дуга является частью области регулярности.

Исходный гамильтониан Стеклова $H$ представим в виде $H=\alpha H_{0}+\beta K_{0}+$ $\gamma f_{1}+\delta f_{2}$, в силу чего при отображении момента изоэнергетические поверхности $Q_{h}^{3}=\{H(x)=h\}$ будут отображаться в сечения бифуркационной диаграммы семейством параллельных прямых $\alpha h+\beta f=c$. При этом следует рассматривать только те прямые, которые пересекают бифуркационную диаграмму по компактному множеству, не проходят через ее особые точки и пересекают дуги диаграммы трансверсально. Тем самым мы гарантируем выполнение условий регулярности, налагаемых на изоэнергетические поверхности.

Несложно заметить, что представленной в этом пункте информации достаточно для того, чтобы определить грубые молекулы этих поверхностей, что составляет грубую лиувиллеву классификацию случая Стеклова. 
После этого для получения инвариантов Фоменко-Цишанга нам остается вычислить числовые метки этих молекул. Однако мы решим более обшую задачу: научимся вычислять меченую молекулу для произвольной допустимой кривой. Под допустимой кривой понимается гладкая кривая, пересекающая дуги бифуркационной диаграммы трансверсально и не проходящая через ее особые точки. Потребуем, как и ранее, чтобы кривая пересекала образ отображения момента по компактному множеству. В конце работы мы предъявим алгоритм вычисления меченой молекулы для произвольной допустимой кривой.

3.4. Классификация "круговых" слоений Лиувилля. Точки $M, N, P$, $Q, R, L$ и $z_{i}$ на рис. 4 являются особыми точками бифуркационной диаграммы. Они образованы точками пересечения, касания или возврата гладких кривых. Рассмотрим произвольную особую точку. Опишем вокруг нее малую окружность. В ее прообразе лежит некоторое трехмерное многообразие, на котором возникает слоение Лиувилля. Инвариант Фоменко-Цишанга этого слоения называют круговой молекулой данной особенности. Оказывается, что, с одной стороны, круговые молекулы много “знают" о глобальном слоении системы, а с другой - их несложно вычислить, так как они целиком определяются локальной структурой слоения вблизи особенности.

Теорема 5. Круговые молекулы всех особых точек случая Стеклова имеют вид, указанный в табличе 2.

Доказательство этой теоремы будет получено в двух следующих пунктах.

3.5. Классификация невырожденных положений равновесия. В прообразах точек $M, N, P, Q, R, L$ бифуркационной диаграммы лежат точки, в которых ранг отображения момента падает до нуля. Это - образы точек положения равновесия динамической системы. Дадим определение невырожденности положения равновесия для интегрируемой гамильтоновой системы с двумя степенями свободы.

Пусть на $\left(M^{4}, \omega\right)$ задана интегрируемая система с гамильтонианом $H$ и дополнительным интегралом $F$, а точка $x \in M^{4}$ такая, что $d H(x)=d F(x)=0$. Тогда на $T_{x} M$ корректно определены два симплектических оператора $A_{H}=\Omega^{-1} d^{2} H$ и $A_{F}=\Omega^{-1} d^{2} F$, порождающие в алгебре Ли $\mathrm{sp}(4, \mathbb{R})$ некоторую коммутативную подалгебру $K(H, F)$.

ОПРЕДЕЛЕНИЕ 9. Положение равновесия $x$ называется невырожденныцм, если подалебра $K(H, F)$ является картановской подалгеброй в $\operatorname{sp}(4, \mathbb{R})$.

Укажем эффективный способ проверки картановости подалгебры $K(H, F)$.

Для начала заметим, что операторы $A_{H}$ и $A_{F}$ совпадают с линеаризациями векторных полей sgrad $H$ и sgrad $F$ соответственно, что позволяет легко вычислять матрицы, которьми они задаются в локальных координатах. Действительно,

$$
\frac{\partial(\operatorname{sgrad} H)^{i}}{\partial x^{j}}=\frac{\partial}{\partial x^{j}}\left(\omega^{i k} \frac{\partial H}{\partial x^{k}}\right)=\omega^{i k} \frac{\partial^{2} H}{\partial x^{j} \partial x^{k}}=\left(\Omega^{-1} d^{2} H\right)_{j}^{i} .
$$

Коммутативная подалгебра в $\mathrm{sp}(4, \mathbb{R})$ является картановской, если и только если она двумерна и среди ее элементов найдется линейньй оператор с попарно раз- 
ТАБЛИЦА 2

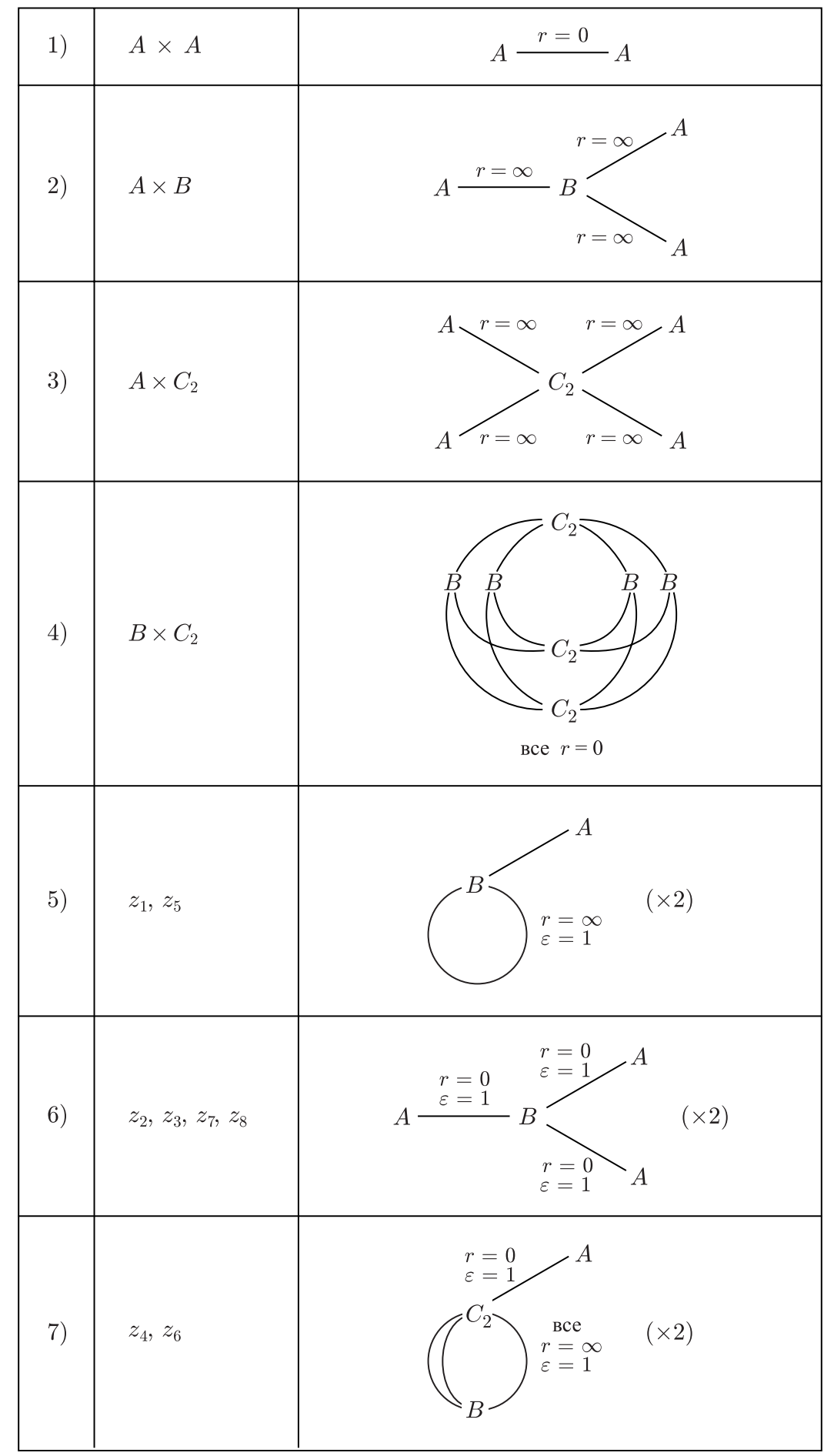


личньми собственными значениями. Итак, сначала нужно убедиться, что операторы $A_{H}$ и $A_{F}$ линейно независимы, и затем проверить, что некоторая линейная комбинация $\lambda A_{H}+\mu A_{F}$ имеет попарно различные собственные значения.

Невырожденные положения равновесия обладают многими замечательньми свойствами. В частности, их окрестности в $M^{4}$ представимы в виде почти прямого произведения 2-атомов [13].

Вернемся к случаю Стеклова.

ТЕОРЕМА 6. Условия невырохсденности, типь и представления в виде почти прямого произведения для положений равновесия, лежащих в прообразах точек $M, N, P, Q, R, L$ бифуркационной диаграммы, указаны в табличе 3.

Круговые молекуль точек $M, N, P, Q, R, L$ указаны в таблице 2.

ТАБЛИЦА 3

\begin{tabular}{|c|c|c|c|}
\hline Точка & Условия невьрожденности & Тип & $\begin{array}{c}\text { Почти прямое } \\
\text { произведение }\end{array}$ \\
\hline$M$ & $\begin{array}{l}g \in\left(-\infty, a_{2}-a_{3}\right) \cup\left(a_{3}-a_{2},+\infty\right) \backslash\left\{-3 a_{1}\right\} \\
g \in\left(a_{2}-a_{3}, a_{3}-a_{2}\right)\end{array}$ & $\begin{array}{l}\text { центр-центр } \\
\text { седло-центр }\end{array}$ & $\begin{array}{l}2(A \times A) \\
2(A \times B)\end{array}$ \\
\hline$N$ & $\begin{array}{l}g \in\left(-\infty, a_{1}-a_{2}\right) \cup\left(a_{2}-a_{1},+\infty\right) \backslash\left\{-3 a_{3}\right\} \\
g \in\left(a_{1}-a_{2}, a_{2}-a_{1}\right)\end{array}$ & $\begin{array}{c}\text { центр-центр } \\
\text { седло-центр }\end{array}$ & $\begin{array}{l}2(A \times A) \\
2(A \times B)\end{array}$ \\
\hline$P$ & $g \in\left(-\infty, a_{1}-a_{3}\right) \cup\left(a_{3}-a_{1},+\infty\right)$ & $\begin{array}{c}\text { седло-центр } \\
\text { седло-седло }\end{array}$ & $\begin{array}{l}\left(A \times C_{2}\right) \\
\left(B \times C_{2}\right)\end{array}$ \\
\hline$Q$ & $g \in\left(a_{1}-a_{3}, a_{3}-a_{1}\right) \backslash\left\{-3 a_{2}\right\}$ & седло-центр & $2\left(A \times C_{2}\right)$ \\
\hline$R$ & $g \in\left(a_{3}-a_{3}, a_{1}-a_{2}\right)$ & седло-центр & $2\left(A \times a_{1}\right)$ \\
\hline$L$ & $g \in\left(a_{2}-a_{3}, a_{2}-a_{1}\right)$ & центр-центр & $4(A \times A)$ \\
\hline
\end{tabular}

ДоказАТЕльство. Сначала докажем невырожденность положений равновесия при указанных значениях $g$. Для этого вычислим матрицы линеаризаций векторных полей $\operatorname{sgrad} H_{0}$ и $\operatorname{sgrad} F_{0}$ в $e(3)^{*}$. Имеем

$$
\begin{aligned}
& \dot{s}_{i}=\left\{s_{i}, H_{0}\right\}, \quad \dot{r}_{i}=\left\{r_{i}, H_{0}\right\} \\
& \Longleftrightarrow\left\{\begin{array}{l}
\dot{s}_{1}=\left(a_{2}-a_{3}\right) s_{2} s_{3}+\left(a_{2}^{2}-a_{3}^{2}\right)\left(r_{2} s_{3}+s_{2} r_{3}\right)+\left(a_{2}^{3}-a_{3}^{3}\right) r_{2} r_{3}, \\
\dot{s}_{2}=\left(a_{3}-a_{1}\right) s_{3} s_{1}+\left(a_{3}^{2}-a_{1}^{2}\right)\left(r_{3} s_{1}+s_{3} r_{1}\right)+\left(a_{3}^{3}-a_{1}^{3}\right) r_{3} r_{1}, \\
\dot{s}_{3}=\left(a_{1}-a_{2}\right) s_{1} s_{2}+\left(a_{1}^{2}-a_{2}^{2}\right)\left(r_{1} s_{2}+s_{1} r_{2}\right)+\left(a_{1}^{3}-a_{2}^{3}\right) r_{1} r_{2}, \\
\dot{r}_{1}=a_{2} s_{2} r_{3}-a_{3} s_{3} r_{2}+\left(a_{2}^{2}-a_{3}^{2}\right) r_{2} r_{3}, \\
\dot{r}_{2}=a_{3} s_{3} r_{1}-a_{1} s_{1} r_{3}+\left(a_{3}^{2}-a_{1}^{2}\right) r_{3} r_{1}, \\
\dot{r}_{3}=a_{1} s_{1} r_{2}-a_{2} s_{2} r_{1}+\left(a_{1}^{2}-a_{2}^{2}\right) r_{1} r_{2} .
\end{array}\right.
\end{aligned}
$$

Дифференцируя правые части уравнений, получаем матрицу линеаризации $A_{H_{0}}^{6}$ векторного поля $\operatorname{sgrad} H_{0}$ в координатах $\left(s_{1}, s_{2}, s_{3}, r_{1}, r_{2}, r_{3}\right)$ :

$$
A_{H_{0}}^{6}=\left(\begin{array}{cc}
A & B \\
C & D
\end{array}\right) \text {, }
$$


где

$$
\begin{aligned}
& A=\left(\begin{array}{ccc}
0 & \left(a_{2}-a_{3}\right) s_{3} & \left(a_{2}-a_{3}\right) s_{2} \\
& +\left(a_{2}^{2}-a_{3}^{2}\right) r_{3} & +\left(a_{2}^{2}-a_{3}^{2}\right) r_{2} \\
\left(a_{3}-a_{1}\right) s_{3} & 0 & \left(a_{3}-a_{1}\right) s_{1} \\
+\left(a_{3}^{2}-a_{1}^{2}\right) r_{3} & & +\left(a_{3}^{2}-a_{1}^{2}\right) r_{1} \\
\left(a_{1}-a_{2}\right) s_{2} & & \\
+\left(a_{1}^{2}-a_{2}^{2}\right) r_{2} & \left(a_{1}-a_{2}\right) s_{1}+\left(a_{1}^{2}-a_{2}^{2}\right) r_{1} & 0
\end{array}\right), \\
& B=\left(\begin{array}{ccc}
0 & \left(a_{2}^{2}-a_{3}^{2}\right) s_{3} & \left(a_{2}^{2}-a_{3}^{2}\right) s_{2} \\
& +\left(a_{2}^{3}-a_{3}^{3}\right) r_{3} & +\left(a_{2}^{3}-a_{3}^{3}\right) r_{2} \\
\left(a_{3}^{2}-a_{1}^{2}\right) s_{3} & & \left(a_{3}^{2}-a_{1}^{2}\right) s_{1} \\
+\left(a_{3}^{3}-a_{1}^{3}\right) r_{3} & 0 & +\left(a_{3}^{3}-a_{1}^{3}\right) r_{1} \\
\left(a_{1}^{2}-a_{2}^{2}\right) s_{2} & \left(a_{1}^{2}-a_{2}^{2}\right) s_{1} & \\
+\left(a_{1}^{3}-a_{2}^{3}\right) r_{2} & +\left(a_{1}^{3}-a_{2}^{3}\right) r_{1} & 0
\end{array}\right) \\
& C=\left(\begin{array}{ccc}
0 & a_{2} r_{3} & -a_{3} r_{2} \\
-a_{1} r_{3} & 0 & a_{3} r_{1} \\
a_{1} r_{2} & -a_{2} r_{1} & 0
\end{array}\right) \\
& D=\left(\begin{array}{ccc}
0 & \left(a_{2}^{2}-a_{3}^{2}\right) r_{3}-a_{3} s_{3} & \left(a_{2}^{2}-a_{3}^{2}\right) r_{2}+a_{2} s_{2} \\
\left(a_{3}^{2}-a_{3}^{1}\right) r_{3}+a_{3} s_{3} & 0 & \left(a_{3}^{2}-a_{1}^{2}\right) r_{1}-a_{1} s_{1} \\
\left(a_{1}^{2}-a_{2}^{2}\right) r_{2}-a_{2} s_{2} & \left(a_{1}^{2}-a_{2}^{2}\right) r_{1}+a_{1} s_{1} & 0
\end{array}\right) .
\end{aligned}
$$

Аналогичные вычисления необходимо проделать для поля $\operatorname{sgrad} F_{0}$ :

$$
\begin{aligned}
& \dot{s}_{i}=\left\{s_{i}, F_{0}\right\}, \quad \dot{r}_{i}=\left\{r_{i}, F_{0}\right\} \\
& \Longleftrightarrow\left\{\begin{array}{l}
\dot{s}_{1}=\left(a_{3}-a_{2}\right)\left(r_{2} s_{3}+s_{2} r_{3}\right)+3\left(a_{3}^{2}-a_{2}^{2}\right) r_{2} r_{3}, \\
\dot{s}_{2}=\left(a_{1}-a_{3}\right)\left(r_{3} s_{1}+s_{3} r_{1}\right)+3\left(a_{1}^{2}-a_{3}^{2}\right) r_{3} r_{1}, \\
\dot{s}_{3}=\left(a_{2}-a_{1}\right)\left(r_{1} s_{2}+s_{1} r_{2}\right)+3\left(a_{2}^{2}-a_{1}^{2}\right) r_{1} r_{2}, \\
\dot{r}_{1}=s_{2} r_{3}-s_{3} r_{2}+\left(a_{3}-a_{2}\right) r_{2} r_{3}, \\
\dot{r}_{2}=s_{3} r_{1}-s_{1} r_{3}+\left(a_{1}-a_{3}\right) r_{3} r_{1}, \\
\dot{r}_{3}=s_{1} r_{2}-s_{2} r_{1}+\left(a_{2}-a_{1}\right) r_{1} r_{2},
\end{array}\right. \\
& A_{F_{0}}^{6}=\left(\begin{array}{ll}
A^{\prime} & B^{\prime} \\
C^{\prime} & D^{\prime}
\end{array}\right), \\
& A^{\prime}=\left(\begin{array}{ccc}
0 & \left(a_{3}-a_{2}\right) r_{3} & \left(a_{3}-a_{2}\right) r_{2} \\
\left(a_{1}-a_{3}\right) r_{3} & 0 & \left(a_{1}-a_{3}\right) r_{1} \\
\left(a_{2}-a_{1}\right) r_{2} & \left(a_{2}-a_{1}\right) r_{1} & 0
\end{array}\right), \\
& B^{\prime}=\left(\begin{array}{ccc}
0 & 3\left(a_{3}^{2}-a_{2}^{2}\right) r_{3} & 3\left(a_{3}^{2}-a_{2}^{2}\right) r_{2} \\
& +\left(a_{3}-a_{2}\right) s_{3} & +\left(a_{3}-a_{2}\right) s_{2} \\
3\left(a_{1}^{2}-a_{3}^{2}\right) r_{3} & & 3\left(a_{1}^{2}-a_{3}^{2}\right) r_{1} \\
+\left(a_{1}-a_{3}\right) s_{3} & 0 & +\left(a_{1}-a_{3}\right) s_{1} \\
3\left(a_{2}^{2}-a_{1}^{2}\right) r_{2} & 3\left(a_{2}^{2}-a_{1}^{2}\right) r_{1} & \\
+\left(a_{2}-a_{1}\right) s_{2} & +\left(a_{2}-a_{1}\right) s_{1} & 0
\end{array}\right)
\end{aligned}
$$




$$
\begin{gathered}
C^{\prime}=\left(\begin{array}{ccc}
0 & r_{3} & -r_{2} \\
-r_{3} & 0 & r_{1} \\
r_{2} & -r_{1} & 0
\end{array}\right), \\
D^{\prime}=\left(\begin{array}{ccc}
0 & \left(a_{3}-a_{2}\right) r_{3}-s_{3} & \left(a_{3}-a_{2}\right) r_{2}+s_{2} \\
\left(a_{1}-a_{3}\right) r_{3}+s_{3} & 0 & \left(a_{1}-a_{3}\right) r_{1}-s_{1} \\
\left(a_{2}-a_{1}\right) r_{2}-s_{2} & \left(a_{2}-a_{1}\right) r_{1}+s_{1} & 0
\end{array}\right) .
\end{gathered}
$$

Рассмотрим точку $M$. В ее прообразе лежат два симметричных положения равновесия $x_{M}= \pm(g, 0,0,1,0,0)$. Для определенности возьмем точку, соответствуюшую знаку “+". Легко убедиться, что в качестве локальных координат в ее 4 -окрестности на $M_{g}^{4}$ можно взять функции $\left(s_{2}, s_{3}, r_{2}, r_{3}\right)$. Касательное пространство к $T_{x_{M}} M_{g}^{4}$ задается уравнениями $\left\{s_{1}=g, r_{1}=1\right\}$. Ограничивая линейные операторы $A_{H_{0}}^{6}$ и $A_{F_{0}}^{6}$ из $e(3)^{*}$ на $T_{x_{M}} M_{g}^{4}$, получаем два симплектических оператора $A_{H_{0}}$ и $A_{F_{0}}$, которые в координатах $\left(s_{2}, s_{3}, r_{2}, r_{3}\right)$ записываются следуюшим образом:

$$
\begin{aligned}
& A_{H_{0}}=\left(\begin{array}{cccc}
0 & \left(a_{3}-a_{1}\right) g & 0 & \left(a_{3}^{2}-a_{1}^{2}\right) g \\
+\left(a_{3}^{2}-a_{1}^{2}\right) & 0 & +\left(a_{3}^{3}-a_{1}^{3}\right) \\
\left(a_{1}-a_{2}\right) g & 0 & \left(a_{1}^{2}-a_{2}^{2}\right) g & 0 \\
+\left(a_{1}^{2}-a_{2}^{2}\right) & 0 & +\left(a_{1}^{3}-a_{2}^{3}\right) & 0 \\
0 & a_{3} & 0 & \left(a_{3}^{2}-a_{1}^{2}\right)-a_{1} g \\
-a_{2} & 0 & \left(a_{1}^{2}-a_{2}^{2}\right)+a_{1} g & 0
\end{array}\right) \\
& A_{F_{0}}=\left(\begin{array}{cccc}
0 & \left(a_{1}-a_{3}\right) & 0 & 3\left(a_{1}^{2}-a_{3}^{2}\right)+\left(a_{1}-a_{3}\right) g \\
a_{2}-a_{1} & 0 & 3\left(a_{2}^{2}-a_{1}^{2}\right)+\left(a_{2}-a_{1}\right) g & 0 \\
0 & 1 & 0 & a_{1}-a_{3}-g \\
-1 & 0 & a_{2}-a_{1}+g & 0
\end{array}\right) .
\end{aligned}
$$

Легко видеть, что эти матрицы линейно независимы при любых $g$ и, тем самым, первое условие невырожденности выполняется. Теперь проверим второе условие. Покажем, что при $g \notin\left\{a_{2}-a_{3}, a_{3}-a_{2},-3 a_{1}, 3 a_{1}\right\}$ матрица $A_{F_{0}}$ имеет различные собственные значения. Эта матрица имеет вид

$$
X=\left(\begin{array}{cccc}
0 & A_{1} & 0 & B_{1} \\
A_{2} & 0 & B_{2} & 0 \\
0 & C_{1} & 0 & D_{1} \\
C_{2} & 0 & D_{2} & 0
\end{array}\right) .
$$

Рассмотрим уравнение на собственные значения:

$$
\begin{aligned}
\operatorname{det}(X-\lambda E)= & \lambda^{4}-\left(A_{1} A_{2}+B_{1} C_{2}+B_{2} C_{1}+D_{1} D_{2}\right) \lambda^{2} \\
& +\left(A_{1} D_{1}-B_{1} C_{1}\right)\left(A_{2} D_{2}-B_{2} C_{2}\right) \\
= & \lambda^{4}-b \lambda^{2}+\Delta_{1} \Delta_{2}=\lambda^{4}-b \lambda^{2}+\Delta=t^{2}-b t+\Delta=0,
\end{aligned}
$$

где

$$
\begin{aligned}
t & =\lambda^{2} \\
b & =A_{1} A_{2}+B_{1} C_{2}+B_{2} C_{1}+D_{1} D_{2}, \\
\Delta & =\Delta_{1} \Delta_{2}=\left(A_{1} D_{1}-B_{1} C_{1}\right)\left(A_{2} D_{2}-B_{2} C_{2}\right) .
\end{aligned}
$$


Для нашего случая имеем

$$
\begin{aligned}
b & =\left(\left(a_{2}-a_{1}\right)\left(a_{1}-4 a_{3}\right)+\left(a_{1}-a_{3}\right)\left(4 a_{2}-a_{1}\right)-g^{2}\right) \\
& =-\left(22 a_{2} a_{3}+7 a_{2}^{2}+7 a_{3}^{2}+g^{2}\right)<0 \\
\Delta & =-4\left(a_{1}-a_{3}\right)\left(a_{2}-a_{1}\right)\left(g^{2}-\left(a_{3}-a_{2}\right)^{2}\right) \\
& =4\left(2 a_{2}^{2}+2 a_{3}^{2}+5 a_{2} a_{3}\right)\left(g^{2}-a_{2}^{2}-a_{3}^{2}+2 a_{2} a_{3}\right) .
\end{aligned}
$$

Вычислим дискриминант квадратного уравнения:

$$
D=\left(22 a_{2} a_{3}+7 a_{2}^{2}+7 a_{3}^{2}+g^{2}\right)^{2}-16\left(2 a_{2}^{2}+2 a_{3}^{2}+5 a_{2} a_{3}\right)\left(g^{2}-a_{2}^{2}-a_{3}^{2}+2 a_{2} a_{3}\right) .
$$

Как видно, дискриминант является квадратичной функцией от $g^{2}$, принимающей положительные значения на $\pm \infty$. Найдем точку минимума и минимальное значение функции $D\left(g^{2}\right)$ :

$$
\begin{aligned}
g_{\min }^{2}= & -\frac{1}{2}\left(2\left(22 a_{2} a_{3}+7 a_{2}^{2}+7 a_{3}^{2}\right)-16\left(2 a_{2}^{2}+2 a_{3}^{2}+5 a_{2} a_{3}\right)\right) \\
= & 9\left(2 a_{2} a_{3}+a_{2}^{2}+a_{3}^{2}\right)=9\left(a_{2}+a_{3}\right)^{2}=\left(3 a_{1}\right)^{2}, \\
D_{\min }= & \left(22 a_{2} a_{3}+7 a_{2}^{2}+7 a_{3}^{2}+9\left(2 a_{2} a_{3}+a_{2}^{2}+a_{3}^{2}\right)\right)^{2} \\
& -16\left(2 a_{2}^{2}+2 a_{3}^{2}+5 a_{2} a_{3}\right)\left(9\left(2 a_{2} a_{3}+a_{2}^{2}+a_{3}^{2}\right)-a_{2}^{2}-a_{3}^{2}+2 a_{2} a_{3}\right) \\
= & 64\left(\left(2 a_{2}^{2}+2 a_{3}^{2}+5 a_{2} a_{3}\right)^{2}-\left(2 a_{2}^{2}+2 a_{3}^{2}+5 a_{2} a_{3}\right)\left(2 a_{2}^{2}+2 a_{3}^{2}+5 a_{2} a_{3}\right)\right)=0 .
\end{aligned}
$$

Корни квадратного уравнения $t_{1}, t_{2} \in \mathbb{R}$, так как $D \geqslant 0$.

При $g \in\left(a_{2}-a_{3}, a_{3}-a_{2}\right)$ имеем $\Delta<0$ и по теореме Виета $t_{2}<0<t_{1}$. Значит, четыре корня $\lambda_{i}$ биквадратного уравнения на собственные значения имеют вид $\left\{ \pm \sqrt{\left|t_{1}\right|}, \pm i \sqrt{\left|t_{2}\right|}\right\}$. Как видно, они попарно различны.

При $g \in\left(-\infty, a_{2}-a_{3}\right) \cup\left(a_{3}-a_{2},+\infty\right)$ имеем $\Delta>0$. С учетом того, что $b<0$, по теореме Виета имеем $t_{1}<t_{2}<0$, а значит, множество собственных значений имеет вид $\left\{ \pm i \sqrt{\left|t_{1}\right|}, \pm i \sqrt{\left|t_{2}\right|}\right\}$, и нужно дополнительно требовать, чтобы $t_{1} \neq t_{2} \Leftrightarrow$ $D \neq 0 \Leftrightarrow g \neq \pm 3 a_{1}$.

Итак, мы доказали, что при $g \notin\left\{a_{2}-a_{3}, a_{3}-a_{2},-3 a_{1}, 3 a_{1}\right\}$ положение равновесия $x_{M}$ в прообразе точки $M$ невырождено. Не приходится сомневаться, что при $g \in\left\{a_{2}-a_{3}, a_{3}-a_{2},-3 a_{1}\right\}$ точка $x_{M}$ действительно вырождается, так как при этих значениях происходят перестройки бифуркационной диаграммы в точке $M$. Однако это не так для $g=3 a_{1}$. На самом деле, при этом $g$ вырождения положения равновесия $x_{M}$ не происходит. Собственные значения матрицы $\left.A_{F_{0}}\right|_{g=3 a_{1}}$ совпадают, но среди линейных комбинаций $\left.\left(\lambda A_{H_{0}}+\mu A_{F_{0}}\right)\right|_{g=3 a_{1}}$ остаются матрицы с попарно различньпи собственными значениями. Для того чтобы в этом убедиться, достаточно проверить, что собственные значения матрицы $\left.A_{H_{0}}\right|_{g=3 a_{1}}$ не совпадают. Действительно, сохраняя введенные обозначения, имеем

$$
\left.A_{H_{0}}\right|_{g=3 a_{1}}=\left(\begin{array}{cccc}
0 & a_{3}^{2}-4 a_{1}^{2} & 0 & a_{3}^{3}-4 a_{1}^{3} \\
& +3 a_{1} a_{3} & & +3 a_{1} a_{3}^{2} \\
4 a_{1}^{2}-a_{2}^{2} & 0 & 4 a_{1}^{3}-a_{2}^{3} & 0 \\
-3 a_{1} a_{2} & 0 & -3 a_{1} a_{2}^{2} & 0 \\
0 & a_{3} & 0 & a_{3}^{2}-4 a_{1}^{2} \\
-a_{2} & 0 & 4 a_{1}^{2}-a_{2}^{2} & 0
\end{array}\right),
$$




$$
\begin{gathered}
-b=\left(a_{2}^{2}+3 a_{1} a_{2}-4 a_{1}^{2}\right)\left(a_{3}^{2}+3 a_{1} a_{3}-4 a_{1}^{2}\right)+a_{3}\left(a_{2}^{3}+3 a_{1} a_{2}^{2}-4 a_{1}^{3}\right) \\
\quad+a_{2}\left(a_{3}^{3}+3 a_{1} a_{3}^{2}-4 a_{1}^{3}\right)+\left(a_{2}^{2}-4 a_{1}^{2}\right)\left(a_{3}^{2}-4 a_{1}^{2}\right) \\
=32 a_{1}^{4}-4 a_{1}^{2}\left(2 a_{2}^{2}+2 a_{3}^{2}+3 a_{1} a_{2}+3 a_{1} a_{3}\right)+2 a_{2}^{2} a_{3}^{2}+6 a_{1} a_{2}^{2} a_{3} \\
\quad+6 a_{1} a_{2} a_{3}^{2}+9 a_{1}^{2} a_{2} a_{3}+a_{2}^{3} a_{3}+a_{2} a_{3}^{3}-4 a_{1}^{3} a_{2}-4 a_{1}^{3} a_{3} \\
=48 a_{1}^{4}+4 a_{1}^{2} a_{2} a_{3}-8 a_{1}^{2} a_{2}^{2}-8 a_{1}^{2} a_{3}^{2}=4 a_{1}^{2}\left(12 a_{1}^{2}+a_{2} a_{3}-2 a_{2}^{2}-2 a_{3}^{2}\right) \\
=4 a_{1}^{2}\left(10 a_{2}^{2}+10 a_{3}^{2}+25 a_{2} a_{3}\right)=20 a_{1}^{2}\left(2 a_{2}^{2}+2 a_{3}^{2}+5 a_{2} a_{3}\right)>0, \\
\Delta=\Delta_{1} \Delta_{2}, \\
\Delta_{2}=\left(a_{3}^{2}+3 a_{1} a_{3}-4 a_{1}^{2}\right)\left(a_{3}^{2}-4 a_{1}^{2}\right)-a_{3}\left(a_{3}^{3}+3 a_{1} a_{3}^{2}-4 a_{1}^{3}\right) \\
=a_{3}^{2}\left(a_{3}^{2}+3 a_{1} a_{3}\right)-4 a_{1}^{2}\left(2 a_{3}^{2}+3 a_{1} a_{3}\right)+16 a_{1}^{4}-a_{3}^{2}\left(a_{3}^{2}+3 a_{1} a_{3}\right)+4 a_{1}^{3} a_{3} \\
=-4 a_{1}^{2}\left(2 a_{3}^{2}+3 a_{1} a_{3}-a_{1} a_{3}\right)+16 a_{1}^{4}=16 a_{1}^{4}-8 a_{1}^{2}\left(a_{3}^{2}+a_{1} a_{3}\right) \\
=8 a_{1}^{2}\left(2 a_{1}^{2}+a_{3} a_{2}\right)=8 a_{1}^{2}\left(2 a_{2}^{2}+2 a_{3}^{2}+5 a_{2} a_{3}\right), \\
\Delta \Delta_{2}=8 a_{1}^{2}\left(2 a_{2}^{2}+2 a_{3}^{2}+5 a_{2} a_{3}\right), \\
\Delta=64 a_{1}^{4}\left(2 a_{2}^{2}+2 a_{3}^{2}+5 a_{2} a_{3}\right)^{2}>0 ; \\
t^{2}-b t+\Delta=0, \\
\frac{D}{4}=\left(\frac{b}{2}\right)^{2}-\Delta=36 a_{1}^{4}\left(2 a_{2}^{2}+2 a_{3}^{2}+5 a_{2} a_{3}\right)^{2}>0, \quad b<0, \quad \Delta>0 \\
\Longrightarrow \quad t_{1}, t_{2} \in \mathbb{R}, \quad t_{2}<t_{1}<0, \quad\left\{\lambda_{i}\right\}=\left\{ \pm i \sqrt{\left|t_{1}\right|}, \pm i \sqrt{\left|t_{2}\right|}\right\} .
\end{gathered}
$$

В прообразах точек $N$ и $P$ бифуркационной диаграммы лежат положения равновесия с координатами $\pm(0,0, g, 0,0,1)$ и $\pm(0, g, 0,0,1,0)$ соответственно. Проверка их условий невырожденности проводится аналогично случаю точки $M$.

Обратимся теперь к точкам $Q, L, R$. В их прообразах лежат по четыре положения равновесия с координатами:

$$
\begin{gathered}
x_{Q}=\left(0,\left(a_{1}-a_{3}\right) u_{1},\left(a_{1}-a_{2}\right) v_{1}, 0, u_{1}, v_{1}\right), \\
u_{1}^{2}=\frac{g+a_{2}-a_{1}}{a_{2}-a_{3}}, \quad v_{1}^{2}=\frac{g+a_{3}-a_{1}}{a_{3}-a_{2}}, \\
a_{1}-a_{3} \leqslant g \leqslant a_{1}-a_{2} ; \\
x_{L}=\left(\left(a_{2}-a_{3}\right) v_{2}, 0,\left(a_{2}-a_{1}\right) u_{2}, v_{2}, 0, u_{2}\right), \\
u_{2}^{2}=\frac{g+a_{3}-a_{2}}{a_{3}-a_{1}}, \quad v_{2}^{2}=\frac{g+a_{1}-a_{2}}{a_{1}-a_{3}}, \\
a_{2}-a_{3} \leqslant g \leqslant a_{2}-a_{1} ; \\
x_{R}=\left(\left(a_{3}-a_{2}\right) u_{3},\left(a_{3}-a_{1}\right) v_{3}, 0, u_{3}, v_{3}, 0\right), \\
u_{3}^{2}=\frac{g+a_{1}-a_{3}}{a_{1}-a_{2}}, \quad v_{3}^{2}=\frac{g+a_{2}-a_{3}}{a_{2}-a_{1}}, \\
a_{3}-a_{2} \leqslant g \leqslant a_{3}-a_{1} .
\end{gathered}
$$

Рассмотрим точку $Q$. Матрипы $A_{H_{0}}$ и $A_{F_{0}}$, записанные в локальных координатах $\left(s_{1}, s_{2}, s_{3}, r_{1}\right)$, имеют вид: 


$$
\begin{aligned}
& A_{H_{0}}=\left(\begin{array}{cccc} 
& \begin{array}{c}
\left(a_{2}-a_{3}\right) \\
\times\left(a_{1}-a_{2}\right) v_{1} \\
+\left(a_{2}^{2}-a_{3}^{2}\right) v_{1}
\end{array} & \begin{array}{c}
\left(a_{2}-a_{3}\right) \\
\times\left(a_{1}-a_{3}\right) u_{1} \\
+\left(a_{2}^{2}-a_{3}^{2}\right) u_{1}
\end{array} & \\
& & & 0 \\
& & 0 & \left(a_{3}^{2}-a_{1}^{2}\right) \\
\left(a_{3}-a_{1}\right) & & & \times\left(a_{1}-a_{2}\right) v_{1} \\
\times\left(a_{1}-a_{2}\right) v_{1} & 0 & & +\left(a_{3}^{3}-a_{1}^{3}\right) v_{1} \\
+\left(a_{3}^{2}-a_{1}^{2}\right) v_{1} & & 0 & \left(a_{1}^{2}-a_{2}^{2}\right) \\
\left(a_{1}-a_{2}\right) & & & \times\left(a_{1}-a_{3}\right) u_{1} \\
\times\left(a_{1}-a_{3}\right) u_{1} & 0 & & +\left(a_{1}^{3}-a_{2}^{3}\right) u_{1} \\
+\left(a_{1}^{2}-a_{2}^{2}\right) u_{1} & & & 0
\end{array}\right), \\
& A_{F_{0}}=\left(\begin{array}{cccc}
0 & \left(a_{3}-a_{2}\right) v_{1} & \left(a_{3}-a_{2}\right) u_{1} & 0 \\
\left(a_{1}-a_{3}\right) v_{1} & 0 & 0 & 3\left(a_{1}^{2}-a_{3}^{2}\right) v_{1} \\
& & & +\left(a_{1}-a_{3}\right)\left(a_{1}-a_{2}\right) v_{1} \\
\left(a_{2}-a_{1}\right) u_{1} & 0 & 0 & 3\left(a_{2}^{2}-a_{1}^{2}\right) u_{1} \\
0 & v_{1} & -u_{1} & +\left(a_{2}-a_{1}\right)\left(a_{1}-a_{3}\right) u_{1}
\end{array}\right) .
\end{aligned}
$$

Они, очевидно, линейно независимы при $v_{1}, u_{1} \neq 0 \Leftrightarrow g \neq\left(a_{1}-a_{3}\right),\left(a_{1}-a_{2}\right)$.

Убедимся, что собственные значения матрицы $A_{F_{0}}$ попарно различны при $g \in$ $\left(a_{1}-a_{3}, a_{1}-a_{2}\right)$. Видно, что, как и в случае точки $M$, матрица распадается и ее определитель $\Delta^{\prime}$ может быть вычислен так:

$$
\begin{aligned}
\Delta_{1}^{\prime} & =2\left(a_{2}-a_{3}\right) u_{1} v_{1}, \\
\Delta_{2}^{\prime} & =u_{1} v_{1}\left(a_{1}-a_{3}\right)\left(a_{2}-a_{1}\right)\left(3\left(a_{2}+a_{1}\right)+\left(a_{1}-a_{3}\right)-3\left(a_{1}+a_{3}\right)+a_{2}-a_{1}\right) \\
& =4\left(a_{1}-a_{2}\right)\left(a_{2}-a_{3}\right)\left(a_{3}-a_{1}\right), \\
\Delta^{\prime} & =\Delta_{1}^{\prime} \Delta_{2}^{\prime}=8 u_{1}^{2} v_{1}^{2}\left(a_{2}-a_{3}\right)^{2}\left(a_{1}-a_{2}\right)\left(a_{3}-a_{1}\right)<0, \quad u_{1}, v_{1} \neq 0 .
\end{aligned}
$$

Уравнение на собственные значения $\lambda_{i}$ матрицы имеет вид:

$$
\begin{gathered}
\lambda^{4}-b^{\prime} \lambda^{2}+\Delta^{\prime}=t^{2}-b^{\prime} t+\Delta^{\prime}=0 \\
\Delta^{\prime}<0 \Rightarrow t_{1}, t_{2} \in \mathbb{R}, t_{2}<0<t_{1} \Rightarrow\left\{\lambda_{i}\right\}=\left\{ \pm \sqrt{\left|t_{1}\right|}, \pm i \sqrt{\left|t_{2}\right|}\right\} .
\end{gathered}
$$

Получаем, что собственные значения попарно различны.

Для точки $R$ в локальных координатах $\left(s_{1}, s_{2}, s_{3}, r_{3}\right)$ получаем

$$
\Delta^{\prime}=8 u_{3}^{2} v_{3}^{2}\left(a_{1}-a_{2}\right)^{2}\left(a_{3}-a_{1}\right)\left(a_{2}-a_{3}\right)<0
$$

при $u_{3}, v_{3} \neq 0$ и все рассуждения будут аналогичны.

Для точки $L$ в локальных координатах $\left(s_{1}, s_{2}, s_{3}, r_{2}\right)$ имеем

$$
\Delta^{\prime \prime}=8 u_{2}^{2} v_{2}^{2}\left(a_{1}-a_{3}\right)^{2}\left(a_{1}-a_{2}\right)\left(a_{2}-a_{3}\right)>0
$$

при $u_{2}, v_{2} \neq 0$ и требуются дополнительные рассуждения. Уравнение на собственные значения $\lambda_{i}$ матрицы имеет вид:

$$
\lambda^{4}-b^{\prime \prime} \lambda^{2}+\Delta^{\prime \prime}=0 .
$$


Проводя соответствующие вычисления, получим:

$$
b^{\prime \prime}=6\left(\left(a_{3}-a_{2}\right) a_{1} u_{2}^{2}+\left(a_{1}-a_{2}\right) a_{3} v_{2}^{2}\right)<0 .
$$

Убедимся, что дискриминант $D^{\prime \prime}$ уравнения

$$
t^{2}-b^{\prime \prime} t+\Delta^{\prime \prime}=0
$$

строго положителен при $g \in\left(a_{2}-a_{3}, a_{2}-a_{1}\right)$. Тогда $t_{1}, t_{2} \in \mathbb{R}$ и по теореме Виета $t_{1}<t_{2}<0$. А значит, множество собственных значений матрицы имеет вид $\left\{ \pm i \sqrt{\left|t_{1}\right|}, \pm i \sqrt{\left|t_{2}\right|}\right\}$. Имеем

$$
\begin{aligned}
\frac{D^{\prime \prime}}{4}= & \left(\frac{b^{\prime \prime}}{2}\right)^{2}-\Delta^{\prime \prime}=9 u_{2}^{4}\left(a_{3}-a_{2}\right)^{2} a_{1}^{2}+9 v_{2}^{4}\left(a_{1}-a_{2}\right)^{2} a_{3}^{2} \\
& +2 u_{2}^{2} v_{2}^{2}\left(a_{1}-a_{2}\right)\left(a_{3}-a_{2}\right)\left(4 a_{1}^{2}+4 a_{3}^{2}+a_{1} a_{3}\right) \\
= & v_{2}^{4}\left(9 t^{2}\left(a_{3}-a_{2}\right)^{2} a_{1}^{2}+2 t\left(a_{1}-a_{2}\right)\left(a_{3}-a_{2}\right)\left(4 a_{1}^{2}+4 a_{3}^{2}+a_{1} a_{3}\right)\right. \\
& \left.+9\left(a_{1}-a_{2}\right)^{2} a_{3}^{2}\right)=\frac{D^{\prime \prime}(t)}{4}, \quad t=\frac{u_{2}^{2}}{v_{2}^{2}}
\end{aligned}
$$

Покажем, что дискриминант $D_{0}$ квадратного трехчлена $D^{\prime \prime}(t) /\left(4 v_{2}^{4}\right)$ строго отрицателен. Тогда из положительности коэффициента при $t^{2}$ будет следовать, что $D^{\prime \prime}(t) / 4>0$. Имеем

$$
\begin{aligned}
\frac{D_{0}}{4} & =\left(a_{1}-a_{2}\right)^{2}\left(a_{3}-a_{2}\right)^{2}\left(\left(4 a_{1}^{2}+4 a_{3}^{2}+a_{1} a_{3}\right)^{2}-81 a_{1}^{2} a_{3}^{2}\right) \\
& =\left(a_{1}-a_{2}\right)^{2}\left(a_{3}-a_{2}\right)^{2} B \\
\frac{B}{16} & =a_{1}^{4}+2 a_{1}^{3} a_{3}-5 a_{1}^{2} a_{3}^{2}+2 a_{1} a_{3}^{3}+a_{3}^{4} \\
& =a_{2}^{2} a_{3}^{2}\left(\left(\frac{a_{1}}{a_{3}}\right)^{2}+2+\left(\frac{a_{3}}{a_{1}}\right)^{2}\right)-7+2\left(\frac{a_{1}}{a_{3}}+\frac{a_{3}}{a_{1}}\right) \\
& =a_{1}^{2} a_{3}^{2}\left(\alpha^{2}+2 \alpha-7\right)<0
\end{aligned}
$$

так как

в силу того, что

$$
\alpha=\frac{a_{1}}{a_{3}}+\frac{a_{3}}{a_{1}} \in(-2.5,-2)
$$

$$
\frac{a_{1}}{a_{3}}=-\frac{a_{2}+a_{3}}{a_{3}}=-\frac{a_{2}}{a_{3}}-1 \in(-2,-1) .
$$

Тем самым, завершена проверка условий невырожденности точек $M, N, P, Q$, $R, L$.

Сложности рассматриваемых особенностей (т.е. число точек положения равновесия на связной компоненте критического слоя) не превосходят двух. В [1] исследованы все невырожденные положения равновесия малой сложности, указаны их типы, приведены представления в виде почти прямого произведения и вычислены соответствующие круговые молекулы. Зная типы регулярных боттовских перестроек и вид бифуркационной диаграммы, несложно определить, какой случай имеет место для каждой конкретной точки. После чего из таблищ, приведенных в [1], получаем необходимую информацию.

Теорема доказана. 
3.6. Круговые молекулы вырожденных одномерных орбит. В особых точках $z_{i}(i=1, \ldots, 8)$ бифуркационной диаграммы $v=\operatorname{sgrad} H_{0} \neq 0$. В их прообразах лежат вырожденные (не боттовские) критические окружности. Установим некоторые общие свойства таких особенностей. Будем предполагать, что все объекты вещественно-аналитические. Отображение момента, как и ранее, будем обозначать через $\mathfrak{F}$. Сформулируем теорему Н. Т. Зунга [14] для нашего случая систем с двумя степенями свободы.

Tеорема 7 (Н.Т. Зунг [14]). Пусть $\operatorname{dim} \mathfrak{F}^{-1}\left(z_{i}\right)=1$ либо $\operatorname{dim} \mathfrak{F}^{-1}\left(z_{i}\right)=2$ и при әтом $\mathfrak{F}^{-1}\left(z_{i}\right)$ содерәсит регулярную точку, тогда в окрестности $U^{4}\left(\mathfrak{F}^{-1}\left(z_{i}\right)\right) \subset M^{4}$ существует симплектическое аналитическое локально свободное действие окружности $S^{1}$. Оно свободно на торах Лиувилля $и$ сохраняет отображсение момента $\mathfrak{F}$.

Рассмотрим “круговое” многообразие $Q_{\tau}^{3}$, лежащее в прообразе малой окружности с центром в особой точке бифуркационной диаграммы. Орбиты $S^{1}$-действия задают на нем структуру расслоения Зейферта, согласованного со слоением Лиувилля. В теореме 3 утверждается, что такое расслоение всегда существует на 3 -атомах. Из теоремы Зунга следует, что расслоения всех 3 -атомов на $Q_{\tau}^{3}$ согласованы и образуют одно глобальное расслоение Зейферта. Важно отметить, что это свойство именно вырожденных одномерных орбит. Для невырожденных положений равновесия этот факт неверен.

Сделаем отсюда выводы о свойствах круговых молекул изучаемых особенностей. В условиях теоремы 7 справедиво

ПРЕДЛОЖЕНИЕ 1. На ребрах, соединяющих два седловъх атома круговой молекуль вырожденной одномерной орбиты, метки $r$ равны $\infty$. На ребрах, соединяющих атом $А$ с седловым, метки $r$ конечны. Все метки в равны +1 .

ДокАЗАТЕЛЬСтво. Хорошо известно, что равенство метки $r$ бесконечности является критерием того, что два седловых атома, которые соединяют рассматриваемое ребро, образуют единое расслоение Зейферта [1]. С другой стороны, минимаксная окружность атома $A$ является слоем этого расслоения. Поэтому стягиваемый цикл атома $A$ не может иметь со слоями расслоения Зейферта нулевой индекс пересечения. Следовательно, метка $r$ ребра “атом $A$-седловой атом" конечна.

Докажем второе утверждение. Равенство метки $\varepsilon=+1$ означает, что критические окружности-траектории двух атомов имеют одинаковую ориентацию с точки зрения обшего расслоения Зейферта. В прообразе точки на гладкой дуге бифуркационной диаграммы может лежать несколько критических окружностей атома. Известно, что в случае топологически устойчивых боттовских перестроек они ориентированы одинаково [1], поэтому далее можно рассматривать лишь одну критическую окружность. Но при движении вдоль гладкой кривой бифуркационной диаграммы и при пересечении особой точки, которая соответствует вырожденной одномерной орбите, эта критическая окру жность непрерывно “пльвет", не меняя своей ориентации, так как $v \neq 0$. Следовательно, ориентации критических окружностей разных атомов также совпадают.

Предложение доказано. 
ЗАмЕчАниЕ 1. Матрица склейки на ребрах "седло-седло" круговой молекулы вырожденной одномерной орбиты всегда равна $\left(\begin{array}{cc}1 & 0 \\ k & -1\end{array}\right)$, где $k \in \mathbb{Z}$. Это следует из определения метки $r=\infty$ и свойств матриц склеек.

ТЕОрема 8. Круговые молекулы особых точек $z_{i}(i=1, \ldots, 8)$ представленыс в табличе 2 .

ДоКАЗАТЕЛЬСтво. Легко видеть, что условия теоремы 7 выполняются для каждой из точек $z_{i}$, поэтому остается вычислить метки $r$ на ребрах, ведущих в атомы $A$. Будем через $r(*-* *)$ обозначать $r$-метку ребра, которое соединяет бифуркации $*$ и $* *$. Рассмотрим ребро круговой молекулы точки $z_{2}$, относящееся к семейству III. Оно соединяет бифуркации $\beta_{1}$ и $\alpha_{5}$. Из круговой молекулы точки $P$ имеем $r\left(\beta_{1}-\gamma_{2}\right)=0$, точки $Q-r\left(\gamma_{2}-\alpha_{5}\right)=\infty$. Следовательно, по правилу сложения меток [1], [7] $r\left(\beta_{1}-\alpha_{5}\right)=0$. Из других семейств торов получаем:

- точка $z_{2}$ :

семейство II: $\quad r\left(\beta_{1}-\gamma_{2}\right)=0, r\left(\gamma_{2}-\alpha_{2}\right)=\infty \Rightarrow r\left(\beta_{1}-\alpha_{2}\right)=0$, семейство II: $\quad r\left(\beta_{1}-\gamma_{1}\right)=0, r\left(\gamma_{1}-\alpha_{4}\right)=\infty \Rightarrow r\left(\beta_{1}-\alpha_{4}\right)=0$;

- точка $z_{3}$ :

семейство I: $\quad r\left(\alpha_{1}-\alpha_{3}\right)=0, r\left(\alpha_{1}-\beta_{2}\right)=\infty \Rightarrow r\left(\beta_{2}-\alpha_{3}\right)=0$, семейство IV: $\quad r\left(\beta_{2}-\gamma_{2}\right)=0, r\left(\gamma_{2}-\alpha_{7}\right)=\infty \Rightarrow r\left(\beta_{2}-\alpha_{7}\right)=0$;

- точка $z_{4}$ :

семейство III: $\quad r\left(\alpha_{6}-\alpha_{5}\right)=0, r\left(\gamma_{2}-\alpha_{5}\right)=\infty \Rightarrow r\left(\gamma_{2}-\alpha_{6}\right)=0$;

- точка $z_{6}$ :

семейство V: $\quad r\left(\alpha_{12}-\alpha_{9}\right)=0, r\left(\gamma_{3}-\alpha_{9}\right)=\infty \Rightarrow r\left(\gamma_{3}-\alpha_{12}\right)=0$;

- точка $z_{7}$ :

семейство II: $\quad r\left(\alpha_{11}-\alpha_{2}\right)=0, r\left(\alpha_{2}-\beta_{3}\right)=\infty \Rightarrow r\left(\beta_{3}-\alpha_{11}\right)=0$, семейство IV: $\quad r\left(\beta_{3}-\gamma_{3}\right)=0, r\left(\gamma_{3}-\alpha_{8}\right)=\infty \Rightarrow r\left(\beta_{3}-\alpha_{8}\right)=0$;

- точка $z_{8}$ :

семейство V: $\quad r\left(\beta_{4}-\gamma_{3}\right)=0, r\left(\gamma_{3}-\alpha_{9}\right)=\infty \Rightarrow r\left(\beta_{4}-\alpha_{9}\right)=0$,

семейство I: $\quad r\left(\beta_{4}-\gamma_{3}\right)=0, r\left(\gamma_{3}-\alpha_{1}\right)=\infty \Rightarrow r\left(\beta_{4}-\alpha_{1}\right)=0$,

семейство I: $\quad r\left(\beta_{4}-\gamma_{1}\right)=0, r\left(\gamma_{1}-\alpha_{10}\right)=\infty \Rightarrow r\left(\beta_{4}-\alpha_{10}\right)=0$.

Осталось вычислить метку $r$ для молекул точек $z_{1}$ и $z_{5}$.

Уравнения, задающие критическое множество в прообразе кривой (2), были найдены А. А. Ошемковьп [9]:

$$
\begin{gathered}
s_{1}=-\left(a_{1}+2 \mu\right) r_{1}, \quad s_{2}=-\left(a_{2}+2 \mu\right) r_{2}, \quad s_{3}=-\left(a_{3}+2 \mu\right) r_{3}, \\
r_{1}^{2}+r_{2}^{2}+r_{3}^{2}=1, \quad s_{1} r_{1}+s_{2} r_{2}+s_{3} r_{3}=g .
\end{gathered}
$$

Эти уравнения определяют двумерную сферу $S^{2} \subset T S^{2}$. В окрестности точки возврата (т.е. при $-g / 3-\delta<\mu<-g / 3+\delta$ ) они задают два несвязных гладких однопараметрических семейства критических окружностей. (Далее будем рассматривать одну компоненту связности.) При переходе через точку возврата окружность в прообразе преврашается из минимаксной в седловую и в точке возврата перестает быть боттовской.

Для определенности рассмотрим достаточно малую окрестность точки $z_{1}$. Мы хотим вычислить метку $r$ на ребре, соединяющем бифуркации $\alpha_{6}$ и $\beta_{1}$. Она определяется индексом пересечения стягиваемого цикла 3 -атома $A$ и цикла, являющегося 
$S^{1}$-слоем прямого произведения, которым образован 3 -атом $B$. Первый цикл - это бесконечно малая окружность в трансверсали к минимаксной критической окружности, а второй цикл - это окружность, проходящая сколь угодно близко от критической седловой окружности. Их индекс пересечения на среднем торе, очевидно, равен 1 , а значит, метка $r=0$.

Теорема доказана.

3.7. Построение допустимых систем координат. Рассмотрим малый отрезок, трансверсально пересекающий дугу бифуркационной диаграммы. В его прообразе лежит многообразие с краем - 3-атом, край которого состоит из некоторого числа (в зависимости от типа бифуркации) граничных торов. Нам предстоит выбрать на каждом таком торе допустимую систему координат в его фундаментальной группе. Однозначно определенный цикл бифуркации * будем обозначать $\lambda_{*}$. В допустимой системе координат 3-атома этой бифуркации он всегда берется за первый элемент базиса. В случае минимаксного атома $A$ за второй элемент берут любой другой цикл, дополняющий первый до базиса. В случае седловых атомов без звездочек, к которым относятся атомы $B$ и $C_{2}$, также требуют, чтобы в совокупности вторые циклы базисов образовывали на 3 -атоме глобальное сечение, над которьм атом представим как тривиальное $S^{1}$-расслоение.

Дополнительно к этому требуется согласованность ориентаций базисов, которая заключается в следуюшем.

Рассмотрим допустимую кривую. В ее прообразе лежит компактная ориентируемая поверхность $X^{3}$. Зафиксируем на ней какую-либо ориентацию. Выберем произвольный интеграл $f$ на $M^{4}$, который был бы монотонен на кривой и коммутировал с гамильтонианом $H_{0}$. Тогда граничные торы всех атомов можно разделить на положительные и отрицательные (по возрастанию $f$ ). Потребуем, чтобы тройка $\left(\lambda_{1}, \lambda_{2}, \operatorname{grad} f\right)$, где $\lambda_{1}, \lambda_{2}$ - соответственно первый и второй элементы базиса на торе, была положительно ориентирована в $X^{3}$, если тор относится к положительной границе атома, и отрицательно ориентирована в противном случае.

Отметим, что, вообще говоря, меченая молекула зависит от выбора ориентации на $X^{3}$, которую мы фиксировали произвольным образом. В результате в ответе возникают две молекулы для каждой поверхности. Они, разумеется, тесно связаны. Правила перестроек молекул при смене ориентации приведены в [1]. Такие молекулы в рамках поставленной задачи различать не принято, так как это не влияет на существование гомеоморфизма, сохраняющего лиувиллево слоение.

Поставим нашей целью предъявить на каждом граничном торе допустимую систему координат, выраженную в однозначно определенных базисных циклах самой этой бифуркации и ее ближайших соседей. Для определенности все атомы будем ориентировать по возрастанию $H_{0}$.

Вначале выберем допустимые координаты на седловых атомах.

Рассмотрим точку $P$ при $a_{1}-a_{3}<g<-3 a_{2}$. Она имеет тип седло-седло. Ее 4-окрестность представима в виде прямого произведения 2-атомов $\left(B \times C_{2}\right)$. Его сомножители изображены на рис. 5 . Граничные циклы атомов $B$ и $C_{2}$ обозначены $u, v_{1}, v_{2}$ и $p_{1}, p_{2}, q_{1}, q_{2}$ соответственно.

Обозначим 3 -атомы, близкие к точке $P$, соответственно $Q_{\gamma_{1}}, Q_{\gamma_{2}}, Q_{\beta_{1}}, Q_{\beta_{3}}$. 

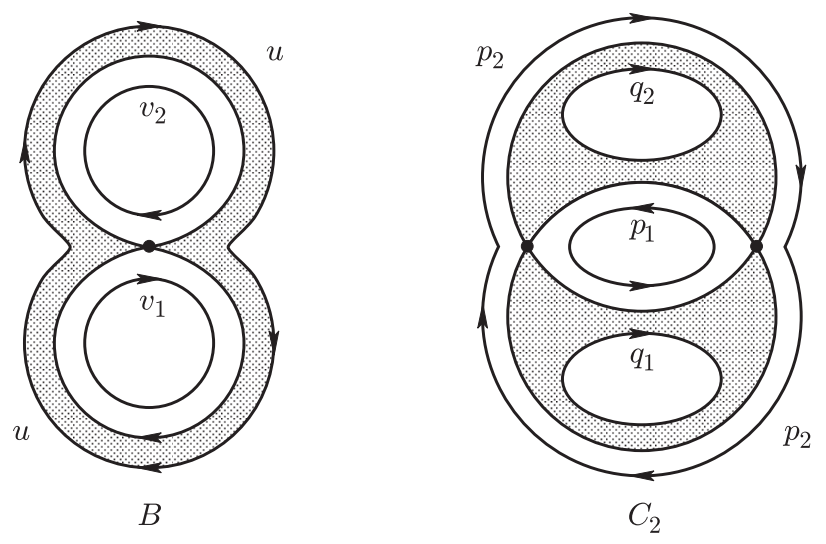

Рис. 5

Тогда имеем

1. $Q_{\gamma_{1}}=C_{2} \times u$;

2. $Q_{\gamma_{2}}=\left(C_{2} \times v_{1}\right) \cup\left(C_{2} \times v_{2}\right)$;

3. $Q_{\beta_{1}}=\left(B \times p_{1}\right) \cup\left(B \times p_{2}\right)$;

4. $Q_{\beta_{2}}=\left(B \times q_{1}\right) \cup\left(B \times q_{2}\right)$.

Вспоминая определение допустимых систем координат на седловых атомах без звездочек, для первого 3 -атома $Q_{\gamma_{1}}$ имеем:

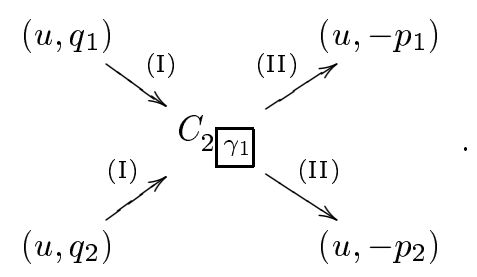

Стрелочки указывают направление роста $H_{0}$. Римские цифры над стрелочками обозначают семейство торов, к которому относится данный граничньй тор. Знак минус требуется для согласования ориентаций систем координат на атоме.

В прообразах остальных трех гладких дуг бифуркационной диаграммы, сходящихся в точке $P$, лежит по два экземпляра соответствующих атомов. Имеем для $i=1,2$ :

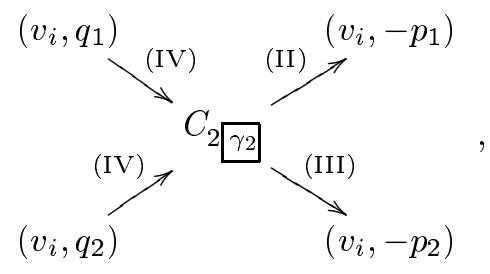




$$
\left(p_{i},-u\right) \stackrel{(\mathrm{II})}{\rightarrow} B
$$

Заметим, что при выборе допустимых систем координат на атоме $Q_{\gamma_{1}}$ мы произвольно поставили знак “-” у правых базисов, а не у левых. Тем самьм, мы фиксировали некоторую ориентацию на многообразии $Q_{\gamma_{1}}^{3}$. Однако после этого альтернативы при выборе допустимых систем координат для остальных трех атомов не возникает: однозначный ответ дает условие согласованности ориентаций базисов, изложенное в начале этого пункта.

Теперь вспомним, что первый базисный цикл бифуркации всегда совпадает с ее однозначно определенньм циклом. Отсюда имеем

$$
\lambda_{\gamma_{1}}=u, \quad \lambda_{\gamma_{2}}=v_{i}, \quad \lambda_{\beta_{1}}=p_{i}, \quad \lambda_{\beta_{2}}=q_{i}
$$

Тем самым, мы выбрали допустимые системы координат, выраженные в однозначно определенных базисных циклах, для дуг $\gamma_{1}, \gamma_{2}, \beta_{1}$ и $\beta_{2}$. Результат выглядит так:

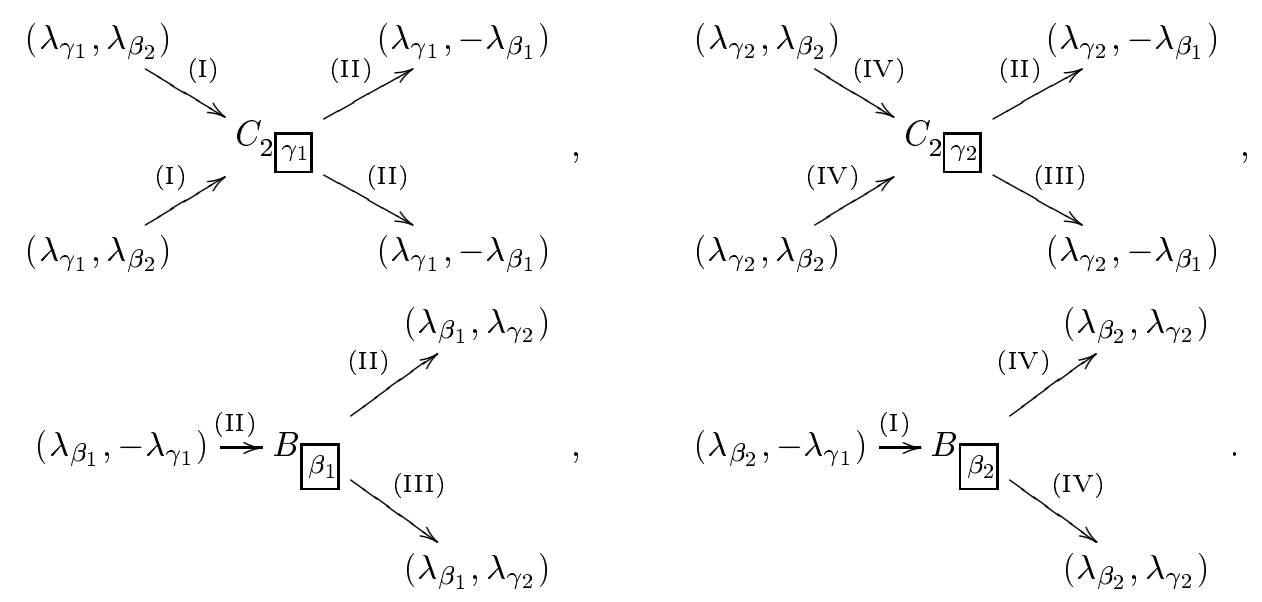

Аналогично, рассмотрев точку $P$ при $-3 a_{2}<g<a_{3}-a_{1}$, получаем допустимые системы координат для бифуркаций $\beta_{3}, \beta_{4}, \gamma_{3}$ и второй вариант допустимых систем координат для $\gamma_{1}$ :

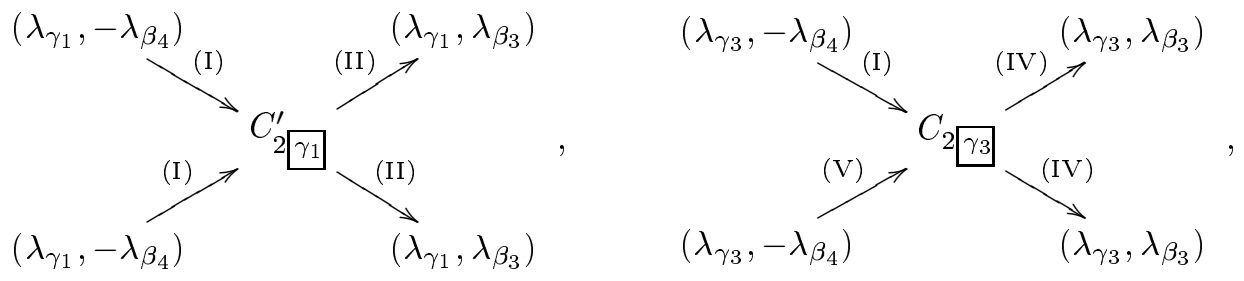



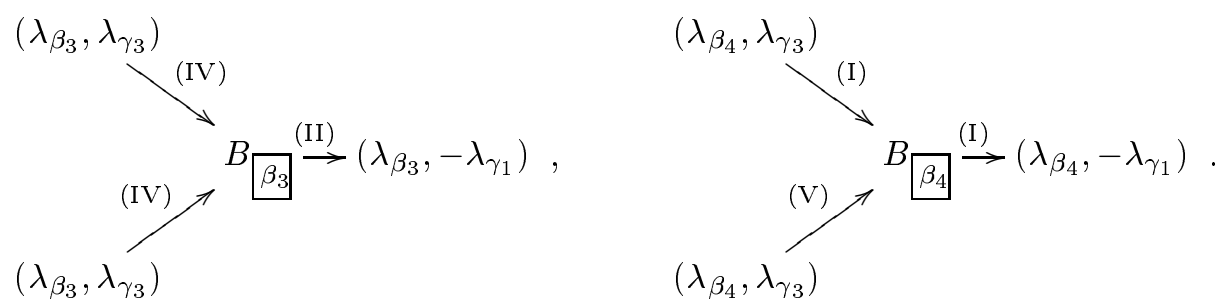

Здесь только необходимо пояснить выбор ориентаций вторых базисных циклов. Напомним, что при выборе допустимых систем координат для бифуркаций $\gamma_{1}, \gamma_{2}$, $\beta_{1}, \beta_{2}$ у нас была альтернатива: взять такие знаки вторых базисных циклов, на которых мы остановились, либо одновременно заменить все знаки на противоположные. Однако после того, как мы фиксировали выбор знаков для бифуркаций $\gamma_{1}, \gamma_{2}, \beta_{1}, \beta_{2}$, аналогичного произвола для дуг $\gamma_{1}, \gamma_{3}, \beta_{3}, \beta_{4}$ уже нет. Покажем, что выбор знаков, указанный вьшше, является единственно верным.

Из круговых молекул особых точек $z_{4}$ и $z_{6}$ следует, что $\lambda_{\gamma_{2}}=\lambda_{\beta_{3}}$ и $\lambda_{\gamma_{3}}=\lambda_{\beta_{4}}$ на торах семейства IV. Обратимся к допустимым базисам бифуркаций $\gamma_{2}$ и $\beta_{3}$, соответствующим последнему семейству. Из структуры слоения вблизи точки $z_{4}$ следует, что они должны быть одинаково ориентированы. Для $\gamma_{2}$ мы выбрали базис $\left(\lambda_{\gamma_{2}}, \lambda_{\beta_{2}}\right)$, поэтому для $\beta_{3}$ следует выбрать базис $\left(\lambda_{\beta_{3}}, \lambda_{\gamma_{3}}\right)$, как и было сделано, а не $\left(\lambda_{\beta_{3}},-\lambda_{\gamma_{3}}\right)$. Таким образом, устраняется произвол в выборе знаков вторых базисных циклов для дуг $\gamma_{1}, \gamma_{3}, \beta_{3}$ и $\beta_{4}$.

Осталось выбрать системы координат на атомах $A$.

Допустимые системы координат на $A$-атомах имеют одно сушественное отличие: регулировка ориентации базиса осуществляется за счет соответствуюшего выбора ориентации первого базисного цикла (он определен однозначно только с точностью до ориентации). И это понятно, так как у атома $A$ второй, а не первый базисный цикл имеет естественную ориентацию, задаваемую гамильтоновым потоком.

Выберем допустимую систему координат для атома $\alpha_{1}$ исходя из круговой молекулы точки $z_{8}$. Метки $r=0$, поэтому пара $\left( \pm \lambda_{\alpha_{1}}, \pm \lambda_{\beta_{4}}\right)$ образует базис на торах семейства I. Осталось разобраться с ориентацией. Перед $\lambda_{\beta_{4}}$ с необходимостью должен стоять знак “+ ", так как на соответствующем ребре круговой молекулы точки $z_{8}$ стоит метка $\varepsilon=+1$. Знак перед $\lambda_{\alpha_{1}}$ лишен смысла, так как этот цикл пока определен с точностью до ориентации. Поставим перед ним знак “+ " , а ориентацию $\lambda_{\alpha_{1}}$ выберем такой, чтобы базис $\left(\lambda_{\alpha_{1}}, \lambda_{\beta_{4}}\right)$ имел такую же ориентацию, как и прочие базисы положительных границ атомов семейства I. Итак,

$$
A_{\alpha_{1}} \stackrel{(\mathrm{I})}{\rightarrow}\left(\lambda_{\alpha_{1}}, \lambda_{\beta_{4}}\right)
$$


Для остальных атомов $A$ из круговых молекул точек $z_{i}$ аналогично получаем:

$$
\begin{aligned}
& \left(\lambda_{\alpha_{2}}, \lambda_{\beta_{1}}\right) \stackrel{(\mathrm{II})}{\rightarrow} A_{\alpha_{2}}, \\
& \left(\lambda_{\alpha_{3}}, \lambda_{\beta_{2}}\right) \stackrel{(\mathrm{I})}{\rightarrow} A_{\alpha_{3}}, \\
& \left(\lambda_{\alpha_{4}}, \lambda_{\beta_{1}}\right) \stackrel{(\mathrm{II})}{\rightarrow} A_{\alpha_{4}}, \\
& \left(\lambda_{\alpha_{5}}, \lambda_{\beta_{1}}\right) \stackrel{(\mathrm{III})}{\rightarrow} A_{\alpha_{5}}, \\
& \left(\lambda_{\alpha_{6}}, \lambda_{\gamma_{2}}\right) \stackrel{(\mathrm{III})}{\rightarrow} A_{\alpha_{6}}, \\
& \left(\lambda_{\alpha_{7}}, \lambda_{\beta_{2}}\right) \stackrel{(\mathrm{IV})}{\rightarrow} A_{\alpha_{7}},
\end{aligned}
$$

$$
A_{\alpha_{8}} \stackrel{(\mathrm{IV})}{\rightarrow}\left(\lambda_{\alpha_{8}}, \lambda_{\beta_{3}}\right)
$$$$
A_{\alpha_{9}} \stackrel{(\mathrm{V})}{\rightarrow}\left(\lambda_{\alpha_{9}}, \lambda_{\beta_{4}}\right) \text {, }
$$$$
A_{\alpha_{10}} \stackrel{(\mathrm{I})}{\rightarrow}\left(\lambda_{\alpha_{10}}, \lambda_{\beta_{4}}\right),
$$$$
A_{\alpha_{11}} \stackrel{(\mathrm{II})}{\rightarrow}\left(\lambda_{\alpha_{11}}, \lambda_{\beta_{3}}\right),
$$$$
A_{\alpha_{12}} \stackrel{(\mathrm{V})}{\rightarrow}\left(\lambda_{\alpha_{12}}, \lambda_{\gamma_{3}}\right)
$$

3.8. Определение взаимного расположения базисных циклов. Проанализируем информацию из круговых молекул и списка допустимых систем координат для определения взаимного расположения циклов $\lambda_{*}$ на торах семейств I-V.

Рассмотрим семейства I. На каждом торе этого семейства бифуркации однозначно определяют циклы $\lambda_{\alpha_{1}}, \lambda_{\alpha_{3}}, \lambda_{\alpha_{10}}, \lambda_{\beta_{2}}, \lambda_{\beta_{4}}, \lambda_{\gamma_{1}}$ и $\lambda_{\gamma_{3}}$. Знаменатель метки $r(*-* *)$ на ребре молекулы равен беззнаковому индексу пересечения циклов $\lambda_{*}$ и $\lambda_{* *}$. Поэтому из круговых молекул особых точек $M, P, R, z_{3}, z_{6}, z_{8}$ можно извлечь информацию об индексах пересечения циклов, которая приведена в таблице 4 .

ТАБЛИЦА 4

\begin{tabular}{|c|c|c|c|}
\hline Пара циклов & $\begin{array}{c}\text { Индекс } \\
\text { пересечения }\end{array}$ & Пара циклов & $\begin{array}{c}\text { Индекс } \\
\text { пересечения }\end{array}$ \\
\hline$\lambda_{\alpha_{1}}$ и $\lambda_{\alpha_{3}}$ & 1 & $\lambda_{\alpha_{1}}$ и $\lambda_{\gamma_{3}}$ & 0 \\
\hline$\lambda_{\alpha_{1}}$ и $\lambda_{\beta_{2}}$ & 0 & $\lambda_{\alpha_{3}}$ и $\lambda_{\gamma_{1}}$ & 0 \\
\hline$\lambda_{\alpha_{10}}$ и $\lambda_{\gamma_{1}}$ & 0 & $\lambda_{\alpha_{3}}$ и $\lambda_{\beta_{2}}$ & 1 \\
\hline$\lambda_{\beta_{2}}$ и $\lambda_{\gamma_{1}}$ & 1 & $\lambda_{\beta_{2}}$ и $\lambda_{\gamma_{3}}$ & 0 \\
\hline$\lambda_{\beta_{4}}$ и $\lambda_{\gamma_{1}}$ & 1 & $\lambda_{\alpha_{1}}$ и $\lambda_{\beta_{4}}$ & 1 \\
\hline$\lambda_{\beta_{4}}$ и $\lambda_{\gamma_{3}}$ & 1 & $\lambda_{\alpha_{10}}$ и $\lambda_{\beta_{4}}$ & 1 \\
\hline
\end{tabular}

С другой стороны, базисы на положительных и отрицательных границах атомов должны иметь разную ориентацию. В силу чего из списка допустимых систем координат следует, что базисы

$$
\left(\lambda_{\alpha_{1}}, \lambda_{\beta_{4}}\right), \quad\left(\lambda_{\alpha_{10}}, \lambda_{\beta_{4}}\right), \quad\left(\lambda_{\gamma_{1}}, \lambda_{\beta_{2}}\right), \quad\left(\lambda_{\gamma_{1}},-\lambda_{\beta_{4}}\right), \quad\left(\lambda_{\gamma_{3}},-\lambda_{\beta_{4}}\right)
$$


должны иметь одинаковую ориентацию, а базисы $\left(\lambda_{\alpha_{3}}, \lambda_{\beta_{2}}\right),\left(\lambda_{\beta_{4}},-\lambda_{\gamma_{1}}\right)$ - противоположную им.

Несложно убедиться, что эти условия определяют взаимное расположение всех циклов рассматриваемого семейства однозначно.

Анализ остальных семейств торов проводится аналогично. Мы не приводим здесь эти рассуждения в силу их тривиальности. В каждом случае информации из списка круговых молекул и допустимых систем координат оказывается достаточно, чтобы однозначно определить взаимное расположение циклов.

На рис. 6 представлен итоговый результат. Фундаментальные группы семейств торов изображены в виде целочисленной решетки на плоскости, а циклы - в виде векторов на ней. Это позволяет наглядно отобразить собранную информацию.

3.9. А лгоритм вычисления инварианта Фоменко-Цишанга. Рисунок 6 следует считать ответом на поставленную в настоящей работе задачу. Действительно, зная взаимное расположение базисных циклов на торах и все допустимые системы координат, можно вычислить молекулу, соответствующую любой допустимой кривой. Действовать следует так:

1. Изобразить кривую на бифуркационной диаграмме.

2. Задать на кривой ориентацию.

3. Выписать допустимые системы координат на атомах, лежащих в прообразе кривой; подкорректировать ориентации атомов, согласуя их с ориентацией на кривой. Напомним, что при смене ориентации на седловых атомах меняются знаки всех вторых базисных циклов, а в случае атома $A$ - знак первого базисного цикла.

4. Выписать матрицы склеек на ребрах молекулы, пользуясь рис. 6.

5. В соответствии с формулами, приведенными в [1; т. 1, гл. 4], вычислить по матрищам склеек числовые метки $r, \varepsilon$ и $n$.

3.10. Пример вычисления меченой молекулы. В качестве примера применения вьшеизложенного алгоритма вычислим метки молекулы, соответствующей вертикальной прямой, проходящей правее всех особых точек бифуркационной диаграммы. Это так называемая молекула больших энергий (для исходного гамильтониана Стеклова $H)$.

Ориентируем прямую по возрастанию $H_{0}$. Ей соответствует грубая молекула:

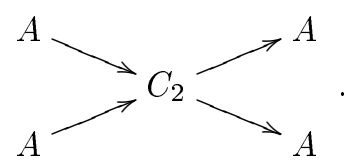

Вьпишем допустимые системы координат атомов молекулы:

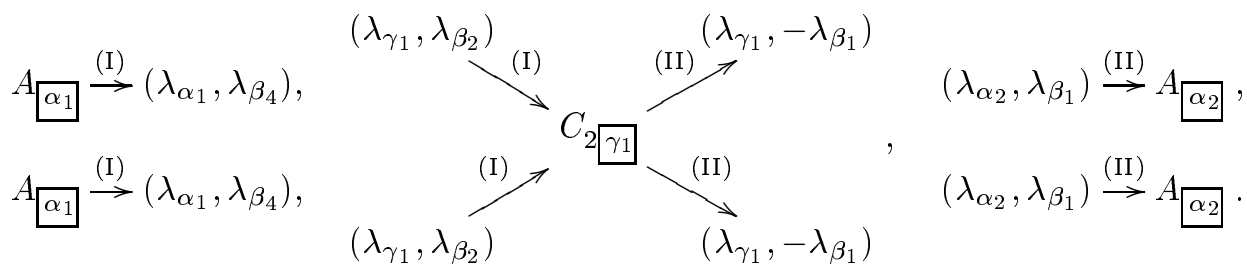




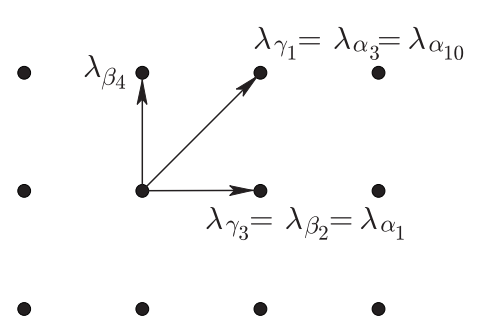

семейство I

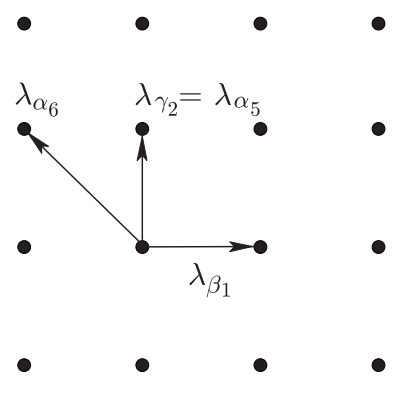

семейство III

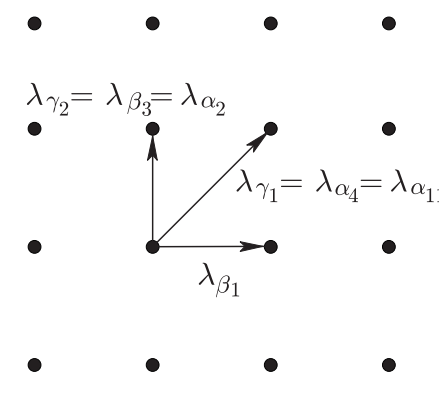

семейство II

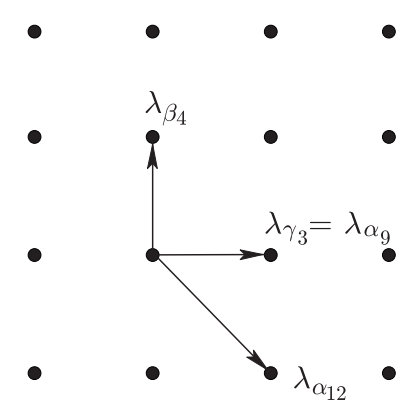

семейство V

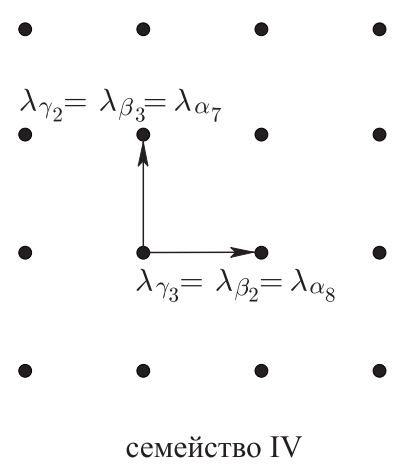

Рис. 6

По рис. 6 вычисляем матрицы склеек:

$$
\left(\begin{array}{l}
\lambda_{\gamma_{1}} \\
\lambda_{\beta_{2}}
\end{array}\right)=\left(\begin{array}{ll}
1 & 1 \\
1 & 0
\end{array}\right)\left(\begin{array}{l}
\lambda_{\alpha_{1}} \\
\lambda_{\beta_{4}}
\end{array}\right), \quad\left(\begin{array}{c}
\lambda_{\alpha_{2}} \\
\lambda_{\beta_{1}}
\end{array}\right)=\left(\begin{array}{rr}
1 & 1 \\
0 & -1
\end{array}\right)\left(\begin{array}{r}
\lambda_{\gamma_{1}} \\
-\lambda_{\beta_{1}}
\end{array}\right)
$$


Фоменко-Цишанга для рассматриваемой прямой:

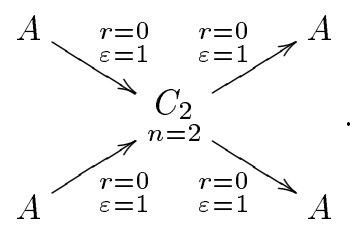

Сопоставляя этот результат с результатами лиувиллевой классификации случая Эйлера [1], можно заключить, что молекулы больших энергий двух случаев совпадают. Этот факт можно также получить, рассматривая случай Стеклова как однопараметрическое возмушение случая Эйлера в классе интегрируемых систем. То, что наши вычисления подтверждают этот вывод, говорит в пользу правильности проделанного анализа.

\section{§4. Лиувиллева классификация интегрируемого случая Соколова}

\section{1. Гамильтониан и дополнительный интеграл случая Соколова.} Интегрируемьй случай Соколова [3], обнаруженный в 2001 году, задается на $e(3)^{*}$ гамильтонианом $H$ :

$$
H=\frac{1}{2}\left(s_{1}^{2}+s_{2}^{2}+2 s_{3}^{2}\right)+r_{2} s_{3}-\frac{1}{2} r_{3}^{2} .
$$

Дополнительный интеграл $F$ четвертой степени имеет вид:

$F=s_{3}^{2}\left(s_{1}^{2}+s_{2}^{2}+s_{3}^{2}+2\left(r_{2} s_{3}-r_{3} s_{2}\right)+r_{2}^{2}+r_{3}^{2}\right)+2 s_{3}\left(s_{2}-r_{3}\right)\left(r_{1} s_{1}+r_{2} s_{2}+r_{3} s_{3}\right)$.

В целом, анализ структуры слоения Лиувилля фазового пространства системы Соколова проводится аналогично случаю Стеклова. Однако здесь метод круговых молекул в чистом виде не дает окончательного ответа, как это было в случае Стеклова, и приходится использовать и другие соображения, например, связанные с формулой Топалова [12].

В отличие от четырехпараметрического случая Стеклова случай Соколова вообше не зависит от параметров. Поэтому для этого случая мы до конца вычислим метки всех изоэнергетических молекул, не останавливаясь на взаимном расположении базисных циклов.

4.2. Результаты П. Е. Рябова. Грубая лиувиллева классификация интегрируемого случая Соколова была получена Рябовым [10]. В этом пункте мы вкратце приводим его результаты.

Теорема 9 (П.Е. Рябов [10]). На плоскости $\mathbb{R}^{2}(f, h)$ бифуркационная диаграмма системы Соколова представляет собой обвединение гладких кривых $\Gamma_{i}, i=1, \ldots, 5$, дде

$\Gamma_{1}: \quad f=-g^{2}, \quad h \geqslant \frac{1}{2}\left(g^{2}-1\right)$,

$\Gamma_{2}: \quad f=2 g^{2} h-g^{4}, \quad \frac{1}{2}\left(g^{2}-1\right) \leqslant h \leqslant g^{2}$,

$\Gamma_{3}: \quad f=\left(h+\frac{1}{2}\right)^{2}-g^{2}, h \geqslant g^{2}-\frac{1}{2}$,

$\Gamma_{4}: f=h^{2}, \quad h \geqslant g^{2}-\frac{1}{4}$,

$\Gamma_{5}: h \geqslant 0$, если $0 \leqslant g \leqslant \frac{1}{2} ; h \geqslant g-\frac{1}{2}$, если $\frac{1}{2} \leqslant g \leqslant 1 ; h \geqslant \frac{g^{2}}{2}$, если $g \geqslant 1$. 

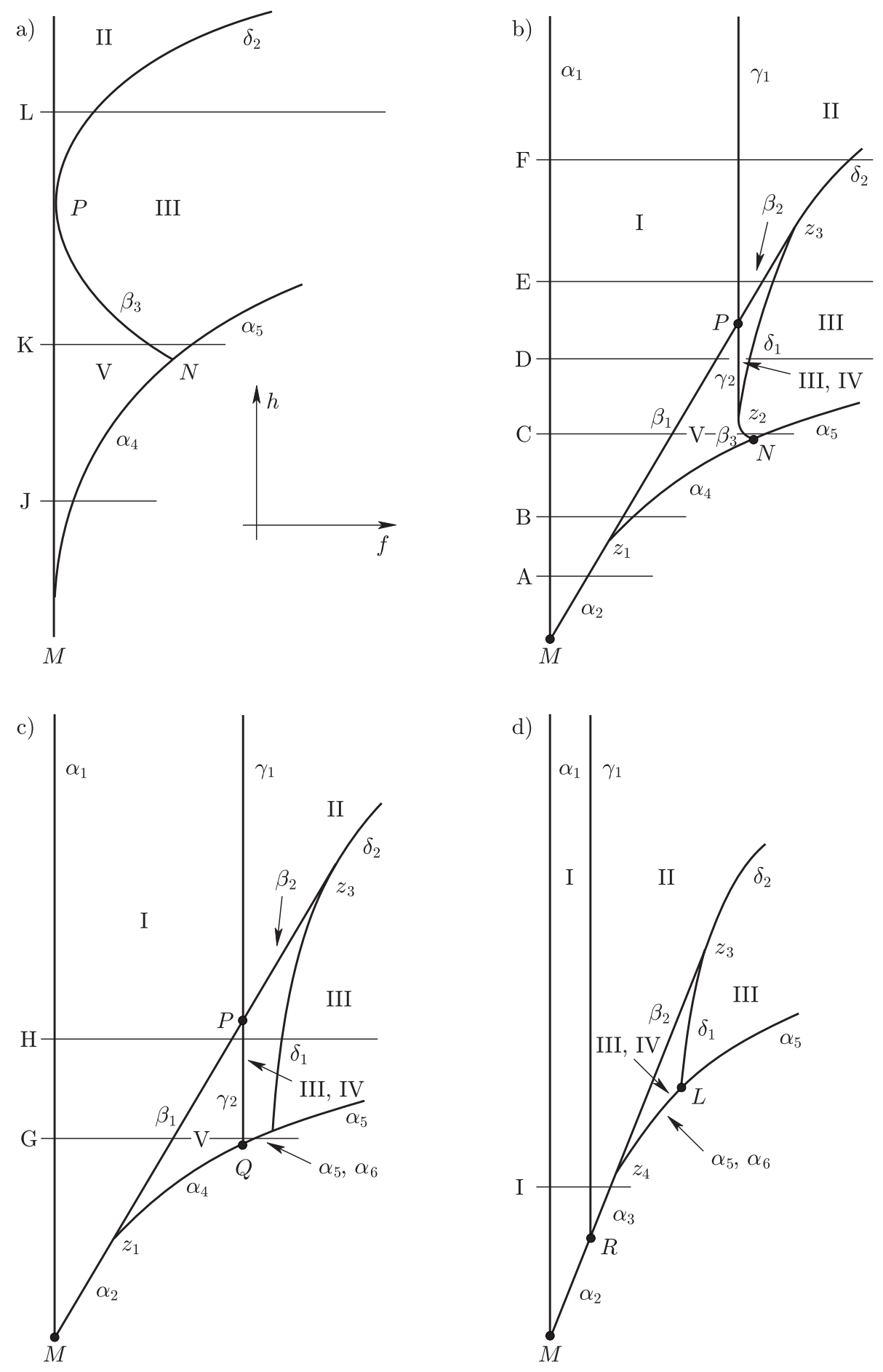

Рис. 7 
В зависимости от значений $g$ имеется четыре качественно различных вида бифуркационной диаграммы (см. рис. 7):

a) $g=0$,

b) $0<g<\frac{1}{2}$,

c) $\frac{1}{2}<g<1$,

d) $g>1$.

Будем далее рассматривать некритические значения $g$. Случай $g=0$ будет разобран отдельно в конще параграфа.

На рис. 7 гладкие дуги бифуркационной диаграммы обозначены малыми греческими буквами с индексами. В их прообразе лежат боттовские перестройки торов Лиувилля, описьваемые 3-атомами. Укажем их типы:

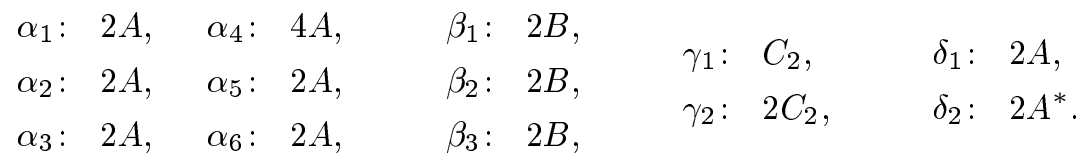

Семейства торов Лиувилля, как и ранее, обозначены римскими цифрами I-V. Каждая точка семейств I-IV образована парой симметричных относительно инволюции $\Phi:(s, r) \rightarrow(-s,-r)$ торов. Семейство $\mathrm{V}$ состоит из четырех компонент связности.

В прообразе прямых A, B, C, D, E, F, G, Н и I лежат изоэнергетические поверхности $Q_{h}^{3}$ с разными типами лиувиллева слоения. П.Е. Рябов [10] указал грубые молекулы, которые им соответствуют, и вычислил топологические типы этих поверхностей. Они таковы: А, В $-2 S^{2}$; C, D, H, I- $S^{1} \times S^{2}$; G $-\left(S^{1} \times S^{2}\right) \#$ $\left(S^{1} \times S^{2}\right) \#\left(S^{1} \times S^{2}\right) ; \mathrm{E}, \mathrm{F}-\mathbb{R} P^{3}$. Нашей задачей является вычисление меток $r, \varepsilon$ и $n$ этих молекул.

4.3. Невырожденные положения равновесия в случае Соколова. В этом пункте мы даем классификацию невырожденных положений равновесия системы Соколова и вычисляем их круговые молекулы.

ТЕОРема 10. Условия невыродсденности, типы и представления в виде почти прямого произведения для положений равновесия, лежащих в прообразах точек $M, N, P, Q, L, R$ бифуркачионной диаграммы, указаны в таблиue 5 .

Круговые молекулы әтих особых точек указаны в табличе 6.

ДокАЗАТЕЛЬСТво. Проверка условий невырожденности положений равновесия случая Соколова осушествляется теми же методами, что и в случае Стеклова. Система уравнений Гамильтона $v=\operatorname{sgrad} H$, записанная в координатах 
ТАБЛИЦА 5

\begin{tabular}{|c|c|c|c|}
\hline Точка & $\begin{array}{c}\text { Условия } \\
\text { невырожденности }\end{array}$ & Тип & $\begin{array}{c}\text { Почти прямое } \\
\text { произведение }\end{array}$ \\
\hline$M$ & $g>0$ & центр-центр & $2(A \times A)$ \\
\hline$N$ & $0<g<\frac{1}{2}$ & седло-центр & $2(A \times B)$ \\
\hline$P$ & $0<g<1$ & седло-седло & $B \times C_{2}$ \\
\hline$Q$ & $\frac{1}{2}<g<1$ & седло-центр & $2\left(A \times C_{2}\right)$ \\
\hline$L$ & $g>\frac{1}{2}$ & центр-центр & $2(A \times A)$ \\
\hline$R$ & $g>1$ & седло-центр & $A \times C_{2}$ \\
\hline
\end{tabular}

ТАБлицА 6

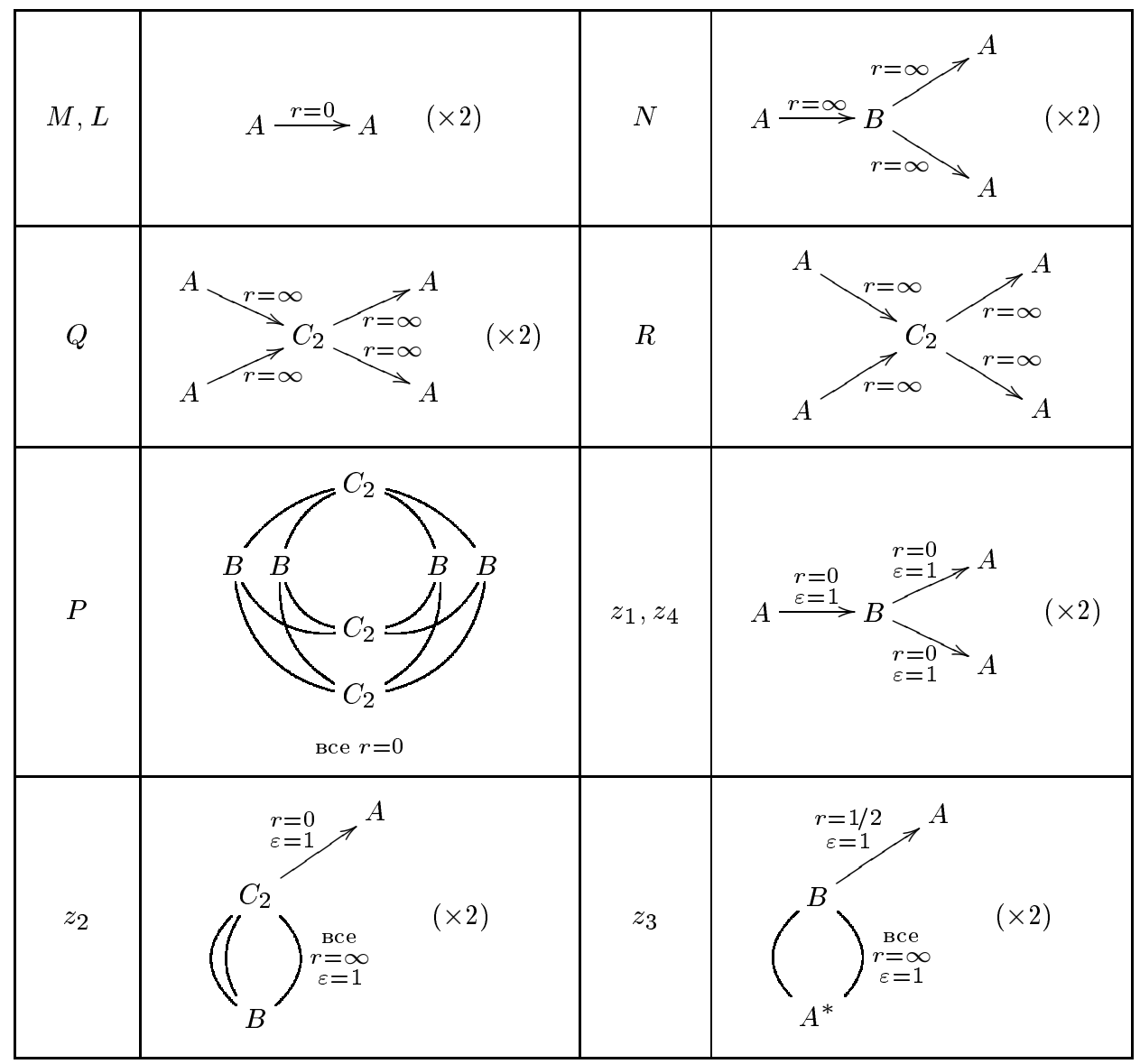


$\left(s_{1}, s_{2}, s_{3}, r_{1}, r_{2}, r_{3}\right)$, имеет вид:

$$
\begin{aligned}
\dot{s}_{i}=\left\{s_{i}, H\right\}, \quad \dot{r}_{i}=\left\{r_{i}, H\right\} \\
\\
\Longleftrightarrow\left\{\begin{array}{l}
\dot{s}_{1}=\left(-s_{2}+r_{3}\right)\left(r_{2}+s_{3}\right), \\
\dot{s}_{2}=s_{3} s_{1}+s_{1} r_{2}-r_{1} r_{3}, \\
\dot{s}_{3}=-r_{1} s_{3}, \\
\dot{r}_{1}=r_{3} s_{2}-2 r_{2} s_{3}-r_{2}^{2}, \\
\dot{r}_{2}=-s_{1} r_{3}+2 r_{1} s_{3}+r_{1} r_{2}, \\
\dot{r}_{3}=s_{1} r_{2}-s_{2} r_{1} .
\end{array}\right.
\end{aligned}
$$

Рассмотрим, к примеру, точки $P$ и $R$. В прообразе каждой лежит по два симметричных положения равновесия с координатами $\pm(g, 0,0,1,0,0)$. Возьмем одну из них. В качестве локальных координат выберем $\left(s_{2}, s_{3}, r_{2}, r_{3}\right)$. На касательном пространстве $T M_{g}^{4}=\left\{s_{1}=g, r_{1}=1\right\}$ получаем оператор:

$$
A_{H}=\left(\begin{array}{cccc}
0 & g & g & -1 \\
0 & -1 & 0 & 0 \\
0 & 1 & 1 & -g \\
-1 & 0 & g & 0
\end{array}\right)
$$

Рассмотрим уравнение на собственные значения:

$\operatorname{det}(A-\lambda E)=(\lambda-1)(\lambda+1)\left(\lambda^{2}+g-1\right)=0 \quad \Longrightarrow \quad \lambda_{1,2}= \pm 1, \quad \lambda_{3,4}= \pm \sqrt{1-g}$.

Как видно, при $g=1$ происходит вырождение: точка $P$ преврашается в точку $R$, меняя свой тип с седло-седло на центр-седло.

Проделаем ту же процедуру для точки $M$. В ее прообразе лежат два положения равновесия:

$$
\pm\left(0, \frac{g^{2}}{\sqrt{g^{2}+1}},-\frac{g}{\sqrt{g^{2}+1}}, 0, \frac{g}{\sqrt{g^{2}+1}},-\frac{1}{\sqrt{g^{2}+1}}\right)
$$

Матрица системы $v=\operatorname{sgrad} H$, линеаризованной в окрестности первой точки с локальными координатами $\left(s_{1}, s_{2}, r_{1}, r_{2}\right)$, имеет вид:

$$
A_{H}=\left(\begin{array}{cccc}
0 & 0 & 0 & -\sqrt{g^{2}+1} \\
\frac{g}{\sqrt{g^{2}+1}} & 0 & \frac{1}{\sqrt{g^{2}+1}} & 0 \\
0 & -\frac{1}{\sqrt{g^{2}+1}} & 0 & \frac{2 g}{\sqrt{g^{2}+1}} \\
\frac{1}{\sqrt{g^{2}+1}} & 0 & -\frac{2 g}{\sqrt{g^{2}+1}} & 0
\end{array}\right) .
$$

Уравнение на собственные значения:

$$
\lambda^{4}+\left(\frac{3 g^{2}}{g^{2}+1}+2\right) \lambda^{2}+\left(\frac{2 g^{2}}{g^{2}+1}+\frac{1}{\sqrt{g^{2}+1}}\right)=0 .
$$


Если положить $t=\lambda^{2}$, то получаем, что при $g>0$ дискриминант

$$
D=\left(\frac{3 g^{2}}{g^{2}+1}\right)^{2}+\frac{4 g^{2}}{g^{2}+1}+4\left(1-\frac{1}{\sqrt{g^{2}+1}}\right)>0 .
$$

Коэффициенты уравнения положительны, поэтому в силу теоремы Виета $t_{2}<$ $t_{1}<0$. А значит, рассматриваемая точка невырождена. Отметим, что $\lambda_{1,2}=$ $\lambda_{3,4}= \pm i$ при $g=0$ и происходит вырождение.

Остальные точки рассматриваются аналогично. Здесь, в отличие от случая Стеклова, это не сопряжено с техническими трудностями, поэтому мы лишь укажем координаты положений равновесия в прообразах этих точек:

$$
\begin{gathered}
N, L: \pm\left(0, \frac{g}{\sqrt{g^{2}+1 / 4}}, \sqrt{g^{2}+\frac{1}{4}}-\frac{1}{2 \sqrt{g^{2}+1 / 4}},\right. \\
\left.0, \frac{1}{2 \sqrt{g^{2}+1 / 4}}, \frac{g}{\sqrt{g^{2}+1 / 4}}\right), \\
Q: \quad \pm(\mp \sqrt{2 g-1}, \sqrt{1-g}, 0, \mp \sqrt{2 g-1}, \sqrt{1-g}, \sqrt{1-g}) .
\end{gathered}
$$

Тем самым, закончена проверка условий невырожденности. Сложности рассматриваемых особенностей не превосходят двух, поэтому типы представления в виде почти прямых произведений и круговые молекулы точек $M, N, P, Q, L$ и $R$ определяются по таблицам, приведенным в [1].

Теорема доказана.

4.4. Круговые молекулы вырожденных одномерных орбит. Точки $z_{1}$, $z_{2}, z_{3}$ и $z_{4}$ соответствуют неботтовским критическим окружностям системы Соколова.

ТЕОРема 11. Круговые молекуль точек $z_{1}, z_{2}, z_{3}$ и $z_{4}$ представлены в табличе 6.

ДокАЗАТЕЛЬСтво. Легко видеть, что условия теоремы 7 для точек $z_{i}$ выполняются, поэтому остается вычислить метки $r$ на ребрах, ведущих в атомы $A$. Будем через $r(*-* *)$ обозначать $r$-метку ребра, которое соединяет бифуркации $*$ и **. Рассмотрим ребра круговой молекулы точки $z_{1}$, относящиеся к семейству V. Они соединяют бифуркации $\beta_{1}$ и $\alpha_{4}$. Из круговой молекулы точки $P$ имеем $r\left(\beta_{1}-\gamma_{2}\right)=0$, точки $Q-r\left(\gamma_{2}-\alpha_{4}\right)=\infty$. Следовательно, $r\left(\beta_{1}-\alpha_{4}\right)=0$. Из других семейств торов получаем:

$$
\begin{array}{ll}
\text { семейство (1): } & r\left(\beta_{1}-\gamma_{1}\right)=0, r\left(\gamma_{1}-\alpha_{2}\right)=\infty \Rightarrow r\left(\beta_{1}-\alpha_{2}\right)=0 ; \\
\text { семейство (2): } & r\left(\beta_{2}-\gamma_{1}\right)=0, r\left(\gamma_{1}-\alpha_{3}\right)=\infty \Rightarrow r\left(\beta_{2}-\alpha_{3}\right)=0 ; \\
\text { семейство (3): } & r\left(\beta_{2}-\gamma_{2}\right)=0, r\left(\gamma_{2}-\alpha_{5}\right)=\infty \Rightarrow r\left(\beta_{2}-\alpha_{5}\right)=0 ; \\
\text { семейство (4): } & r\left(\beta_{2}-\gamma_{2}\right)=0, r\left(\gamma_{2}-\alpha_{6}\right)=\infty \Rightarrow r\left(\beta_{2}-\alpha_{6}\right)=0 ; \\
\text { семейство (5): } & r\left(\delta_{1}-\alpha_{6}\right)=0, r\left(\gamma_{2}-\alpha_{6}\right)=\infty \Rightarrow r\left(\gamma_{2}-\alpha_{6}\right)=0 .
\end{array}
$$

В результате невычисленной остается только метка $r$ на ребре $A-B$ молекулы точки $z_{3}$. В действительности, она равна $\frac{1}{2}$. Этот факт мы получим, вычислив изоэнергетические молекулы. Здесь мы приводим его для полноты картины.

Теорема доказана. 
4.5. Допустимые системы координат в случае Соколова. Допустимые системы координат на 3 -атомах кривых $\beta_{1}, \beta_{2}, \gamma_{1}, \gamma_{2}$ выбираются исходя из 4-окрестности точки $P$, которая имеет тип прямого произведения $B \times C_{2}$. (Стрелочки указывают направление роста $F$.)

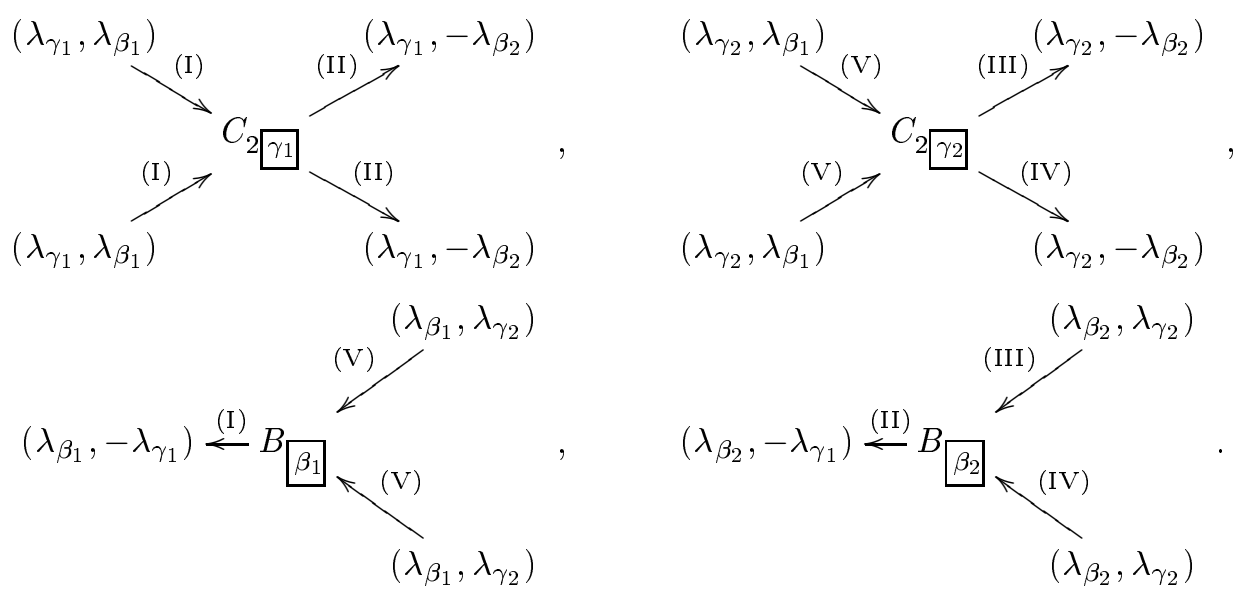

Допустимые системы координат на атомах $A$ выберем, пользуясь тем, что на соответствуюших ребрах круговых молекул точек $M, z_{1}, z_{2}, z_{4} r$-метки равны нулю. Получаем

$$
\begin{aligned}
& A_{\alpha_{1}} \stackrel{(\mathrm{I})}{\rightarrow}\left(\lambda_{\alpha_{1}}, \lambda_{\alpha_{2}}\right), \\
& \left(\lambda_{\alpha_{2}}, \lambda_{\beta_{1}}\right) \stackrel{(\mathrm{I})}{\rightarrow} A \alpha_{\alpha_{2}}, \\
& \left(\lambda_{\alpha_{5}}, \lambda_{\beta_{2}}\right) \stackrel{(\mathrm{III})}{\rightarrow} A_{\alpha_{5}}, \\
& \left(\lambda_{\alpha_{3}}, \lambda_{\beta_{2}}\right) \stackrel{(\mathrm{II})}{\rightarrow} A_{\alpha_{3}}, \\
& \left(\lambda_{\alpha_{6}}, \lambda_{\beta_{2}}\right) \stackrel{(\mathrm{IV})}{\rightarrow} A_{\alpha_{6}}, \\
& \left(\lambda_{\alpha_{4}}, \lambda_{\beta_{1}}\right) \stackrel{(\mathrm{V})}{\rightarrow} A_{\alpha_{4}}, \\
& \left(\lambda_{\delta_{1}}, \lambda_{\gamma_{2}}\right) \stackrel{(\mathrm{IV})}{\rightarrow} A_{\delta_{1}} .
\end{aligned}
$$

Мы не будем выбирать вторые базисные циклы для атомов $B \beta_{\beta_{3}}^{\text {и }} A_{\left[\delta_{2}\right.}^{*}$ в терминах $\lambda_{*}$. Обозначим их $x_{\beta},-y_{\beta},-x_{\delta}, y_{\beta}$. Тогда

$$
\begin{aligned}
& \left(\lambda_{\beta_{3}}, x_{\beta}\right) \\
& \left(\lambda_{\delta_{3}}, x_{\beta}\right) \\
& \stackrel{(\mathrm{III})}{\longrightarrow} A_{\delta_{2}}^{*} \stackrel{(\mathrm{III})}{\longrightarrow}\left(\lambda_{\beta_{3}}, y_{\beta}\right) .
\end{aligned}
$$


Из замечания к предложению 1 следует, что

$$
\left\{\begin{array}{l}
x_{\beta}=\lambda_{\beta_{1}}+k_{1} \lambda_{\gamma_{2}}, \\
y_{\beta}=\lambda_{\beta_{2}}+k_{2} \lambda_{\gamma_{2}}, \\
x_{\delta}=\lambda_{\gamma_{2}}+k_{3} \lambda_{\delta_{2}}, \\
y_{\delta}=\lambda_{\gamma_{1}}+k_{4} \lambda_{\delta_{2}},
\end{array}\right.
$$

где $k_{i} \in \mathbb{Z}$ неизвестны.

4.6. Определение взаимного расположения базисных циклов. Проанализируем информацию из круговых молекул и списка допустимых систем координат для определения взаимного расположения циклов $\lambda_{*}$. Будем рассматривать семейства торов Лиувилля I-V поочередно.

Семейство І. На каждом торе этого семейства бифуркации однозначно определяют циклы $\lambda_{\alpha_{1}}, \lambda_{\alpha_{2}}, \lambda_{\beta_{1}}, \lambda_{\gamma_{1}}$. Знаменатель метки $r(*-* *)$ на ребре молекулы равен беззнаковому индексу пересечения циклов $\lambda_{*}$ и $\lambda_{* *}$. Поэтому из круговых молекул особых точек $M, P, R, z_{1}$ можно заключить, что

- индекс пересечения циклов $\lambda_{\alpha_{1}}$ и $\lambda_{\alpha_{2}}$ равен 1 ,

- индекс пересечения циклов $\lambda_{\beta_{1}}$ и $\lambda_{\gamma_{1}}$ равен 1 ,

- индекс пересечения циклов $\lambda_{\alpha_{2}}$ и $\lambda_{\gamma_{1}}$ равен 0 ,

- индекс пересечения циклов $\lambda_{\alpha_{2}}$ и $\lambda_{\beta_{1}}$ равен 1 .

С другой стороны, базисы на положительных и отрицательных границах атомов должны иметь разную ориентацию. В силу этого из списка допустимых систем координат следует, что базисы $\left(\lambda_{\alpha_{2}}, \lambda_{\beta_{1}}\right),\left(\lambda_{\gamma_{1}}, \lambda_{\beta_{1}}\right),\left(\lambda_{\beta_{1}},-\lambda_{\gamma_{1}}\right)$ должны иметь одинаковую ориентацию, а базис $\left(\lambda_{\alpha_{1}}, \lambda_{\alpha_{2}}\right)$ - противоположную им.

Несложно убедиться, что эти условия определяют взаимное расположение циклов $\lambda_{\alpha_{1}}, \lambda_{\alpha_{2}}$ и $\lambda_{\gamma_{1}}$ однозначно, а про цикл $\lambda_{\beta_{1}}$ можно утверждать, что

$$
\lambda_{\beta_{1}}=\lambda_{\alpha_{1}}+l_{1} \lambda_{\gamma_{1}}, \quad l_{1} \in \mathbb{Z}
$$

На рис. 8 фундаментальная группа тора изображена в виде целочисленной решетки на плоскости, а циклы - в виде векторов на ней. Это позволяет наглядно отобразить собранную информацию.

Семейство II. Циклы семейства: $\lambda_{\alpha_{3}}, \lambda_{\beta_{2}}, \lambda_{\gamma_{1}}, \lambda_{\delta_{2}}$.

Индексы пересечения циклов:

\begin{tabular}{|l|l|}
\hline$\lambda_{\beta_{2}}$ и $\lambda_{\delta_{2}}$ & 0 \\
\hline$\lambda_{\beta_{2}}$ и $\lambda_{\gamma_{1}}$ & 1 \\
\hline$\lambda_{\alpha_{3}}$ и $\lambda_{\gamma_{1}}$ & 0 \\
\hline$\lambda_{\alpha_{3}}$ и $\lambda_{\beta_{2}}$ & 1 \\
\hline
\end{tabular}




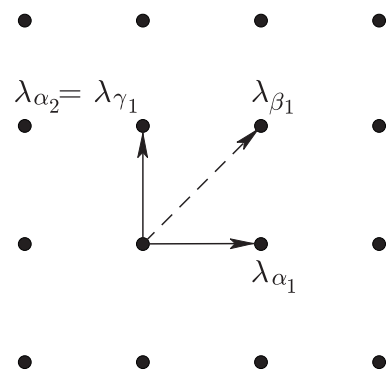

семейство I
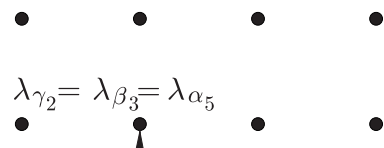

$\bullet$

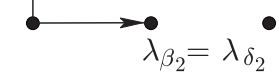

семейство III

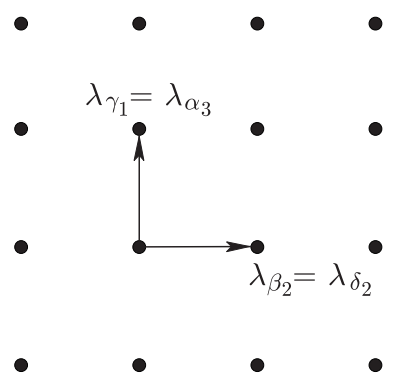

семейство II

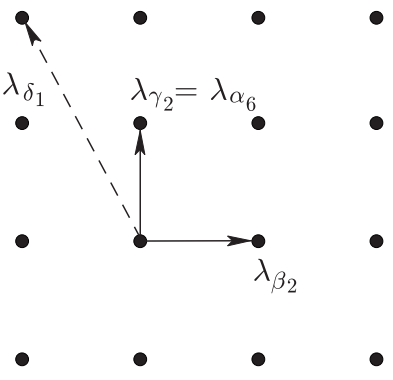

семейство IV

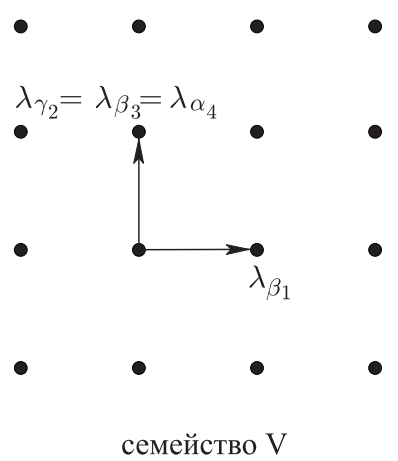

Рис. 8

Из меток $\varepsilon$ круговой молекулы точки $z_{3}$ следует, что $\lambda_{\beta_{2}}=\lambda_{\delta_{2}}$.

При этом базисы $\left(\lambda_{\alpha_{3}}, \lambda_{\beta_{2}}\right),\left(\lambda_{\beta_{2}}, \lambda_{\gamma_{1}}\right)$ имеют одинаковую ориентацию.

Перечисленные условия однозначно определяют взаимное расположение циклов $\lambda_{*}$ этого семейства. Результат изображен на рис. 2.

Семейство III. Циклы семейства: $\lambda_{\alpha_{5}}, \lambda_{\beta_{2}}, \lambda_{\beta_{3}}, \lambda_{\gamma_{2}}, \lambda_{\delta_{2}}$. 
Индексы пересечения циклов:

\begin{tabular}{|l|l|}
\hline$\lambda_{\beta_{2}}$ и $\lambda_{\delta_{2}}$ & 0 \\
\hline$\lambda_{\beta_{2}}$ и $\lambda_{\gamma_{2}}$ & 1 \\
\hline$\lambda_{\alpha_{5}}$ и $\lambda_{\gamma_{2}}$ & 0 \\
\hline$\lambda_{\alpha_{5}}$ и $\lambda_{\beta_{2}}$ & 1 \\
\hline
\end{tabular}

Из круговой молекулы точек $z_{2}$ и $z_{3}$ следует, что $\lambda_{\beta_{2}}=\lambda_{\delta_{2}}$ и $\lambda_{\beta_{3}}=\lambda_{\gamma_{2}}$.

При этом базисы $\left(\lambda_{\alpha_{5}}, \lambda_{\beta_{2}}\right)$ и $\left(\lambda_{\beta_{2}}, \lambda_{\gamma_{2}}\right)$ имеют противоположную ориентацию. Перечисленные условия однозначно определяют взаимное расположение циклов $\lambda_{*}$ этого семейства. Результат изображен на рис. 2.

Семейство IV. Циклы семейства: $\lambda_{\alpha_{6}}, \lambda_{\beta_{2}}, \lambda_{\gamma_{2}}, \lambda_{\delta_{1}}$.

Индексы пересечения циклов:

\begin{tabular}{|c|c|}
\hline$\lambda_{\gamma_{2}}$ и $\lambda_{\delta_{1}}$ & 1 \\
\hline$\lambda_{\beta_{2}}$ и $\lambda_{\gamma_{2}}$ & 1 \\
\hline$\lambda_{\alpha_{6}}$ и $\lambda_{\gamma_{2}}$ & 0 \\
\hline$\lambda_{\alpha_{6}}$ и $\lambda_{\delta_{1}}$ & 1 \\
\hline$\lambda_{\alpha_{6}}$ и $\lambda_{\beta_{2}}$ & 1 \\
\hline
\end{tabular}

При этом базисы $\left(\lambda_{\alpha_{6}}, \lambda_{\beta_{2}}\right),\left(\lambda_{\delta_{1}}, \lambda_{\gamma_{2}}\right)$ имеют одинаковую ориентацию, а базис $\left(\lambda_{\beta_{2}}, \lambda_{\gamma_{2}}\right)$ - противоположную им.

Перечисленные условия однозначно определяют взаимное расположение циклов $\lambda_{\alpha_{6}}, \lambda_{\beta_{2}}, \lambda_{\gamma_{2}}$. Про цикл $\lambda_{\delta_{1}}$ можно утверждать, что

$$
\lambda_{\delta_{1}}=-\lambda_{\beta_{2}}+l_{2} \lambda_{\gamma_{2}}, \quad l_{2} \in \mathbb{Z}
$$

Более того, на ребре $B-A$ молекулы точки $z_{3}$ метка $r$ конечна, а метка $\varepsilon=+1$, поэтому с необходимостью вьполнено $l_{2} \geqslant 1$.

Семейство V. Циклы семейства: $\lambda_{\alpha_{4}}, \lambda_{\beta_{1}}, \lambda_{\beta_{3}}, \lambda_{\gamma_{2}}$.

Индексы пересечения циклов:

\begin{tabular}{|l|l|}
\hline$\lambda_{\beta_{3}}$ и $\lambda_{\gamma_{2}}$ & 0 \\
\hline$\lambda_{\beta_{1}}$ и $\lambda_{\gamma_{2}}$ & 1 \\
\hline$\lambda_{\alpha_{4}}$ и $\lambda_{\gamma_{2}}$ & 0 \\
\hline$\lambda_{\alpha_{4}}$ и $\lambda_{\beta_{1}}$ & 1 \\
\hline
\end{tabular}


Из круговой молекулы точки $z_{2}$ следует, что $\lambda_{\beta_{3}}=\lambda_{\gamma_{2}}$.

При этом базисы $\left(\lambda_{\alpha_{4}}, \lambda_{\beta_{1}}\right)$ и $\left(\lambda_{\gamma_{2}}, \lambda_{\beta_{1}}\right)$ имеют одинаковую ориентацию.

Перечисленные условия однозначно определяют взаимное расположение циклов $\lambda_{*}$ этого семейства. Результат изображен на рис. 8.

4.7. Применение формулы Топалова. Результаты двух предыдущих пунктов позволяют выписать матрицы склеек изоэнергетических молекул с неизвестньми коэффициентами $k_{1}, k_{2}, k_{3}, k_{4}, l_{1}, l_{2}$. Они приведены в таблице 6 . Оказывается, что для того, чтобы вычислить по этим матрицам метки $r, \varepsilon$ и $n$, вовсе не обязательно знать точные значения всех коэффициентов. Легко видеть, что от $k_{1}$ и $k_{2}$ метки вообше не зависят, а от $k_{3}$ и $k_{4}$ зависит только $n$-метка на атомах $A^{*}$ молекулы F.

ПрЕДЛОЖЕНИЕ 2. На атомах $A^{*}$ в молекуле $\mathrm{F}$ метка $n=-1 ; l_{1}=1$, $l_{2}=2$.

ДокАЗАТЕЛЬСТво. Будем далее коэффициенты матриц склеек обозначать следующим образом:

$$
C=\left(\begin{array}{ll}
\alpha & \beta \\
\gamma & \delta
\end{array}\right)
$$

Пусть некоторая молекула $W^{*}$ имеет вид дерева и не содержит семей из более чем одного атома. Пусть также все атомы $W^{*}$ имеют род 0 . Тогда энергия молекулы $N\left(W^{*}\right)$, введенная П. Топаловым [12], определяется формулой:

$$
N\left(W^{*}\right)= \begin{cases}0, & \text { если } \operatorname{rk} H_{1}\left(Q^{3}\right)>0 \\ \mid \text { Tor } H_{1}\left(Q^{3}\right) \mid, & \text { если } \operatorname{rk} H_{1}\left(Q^{3}\right)=0 .\end{cases}
$$

Определим для каждой семьи число $\widetilde{n}=n+\sum r_{i}+p / 2$, где $n-$ ее $n$-метка, $r_{i}-$ метки $r$ на ее внешних ребрах, а $p$ - число звездочек данной семьи. П. Топалов установил формулу, связываюшую $N\left(W^{*}\right)$ с метками молекулы $W^{*}$. Для молекул $\mathrm{E}, \mathrm{F}$ она имеет вид:

$$
N\left(W^{*}\right)= \pm 2^{\sum p}\left(\widetilde{n}_{1} \widetilde{n}^{2}-2 \widetilde{n} \beta_{0}^{-2}\right) \prod_{j} \beta_{j},
$$

где число $\widetilde{n}_{1}$ соответствует атому $C_{2}, \widetilde{n}$ - атомам $B$ или $A^{*}, \beta_{0}$ - коэффициент матрицы склейки на ребрах $C_{2}-B$ либо $C_{2}-A^{*}$, а произведение $\prod_{j} \beta_{j}$ берется по всем ребрам молекулы.

Нам известно, что для молекул Е и $\mathrm{F}$

$$
Q^{3} \cong \mathbb{R} P^{3} \quad \Rightarrow \quad H_{1}\left(Q^{3}\right)=\mathbb{Z}_{2} \quad \Rightarrow \quad N\left(W^{*}\right)=2
$$

Для молекулы Е имеем $n(B)=\left[-1 / l_{2}\right]=-1$ (ведь $\left.l_{2} \geqslant 1\right), \widetilde{n}_{1}=-2 l_{1}$. Формула Топалова дает:

$$
\pm 2=l_{2}^{2}\left(-2 l_{1}\left(-1+1-\frac{1}{l_{2}}\right)^{2}-2\left(-1+1-\frac{1}{l_{2}}\right)\right) \Leftrightarrow l_{1}= \pm 1+l_{2} \quad \Rightarrow \quad l_{1} \geqslant 0 .
$$


Для молекулы $\mathrm{F} \widetilde{n}=n\left(A^{*}\right)+1 / 2, \widetilde{n}_{1}=-2 l_{1}$. Получаем

$$
\begin{aligned}
& \pm 2=4\left(-2 l_{1}\left(n\left(A^{*}\right)+\frac{1}{2}\right)^{2}-2\left(n\left(A^{*}\right)+\frac{1}{2}\right)\right), \\
& \pm 2=\left(-2 l_{1}\left(2 n\left(A^{*}\right)+1\right)^{2}-4\left(2 n\left(A^{*}\right)+1\right)\right), \\
& \pm 1=\left(2 n\left(A^{*}\right)+1\right)\left(l_{1}\left(2 n\left(A^{*}\right)+1\right)+2\right) .
\end{aligned}
$$

При условии $l_{1} \geqslant 0$ это уравнение в целых числах имеет единственное решение $n\left(A^{*}\right)=-1, l_{1}=1 \Rightarrow l_{2}=2$. Что завершает доказательство.

Теперь по таблице 7 можно вычислить все метки изоэнергетических молекул $\mathrm{A}, \ldots, \mathrm{I}$.

ЗАмечаниЕ 2. Из молекулы Е, в частности, следует, что конечная метка $r=1 / 2$ в круговой молекуле точки $z_{3}$.

ТАБЛИЦА 7

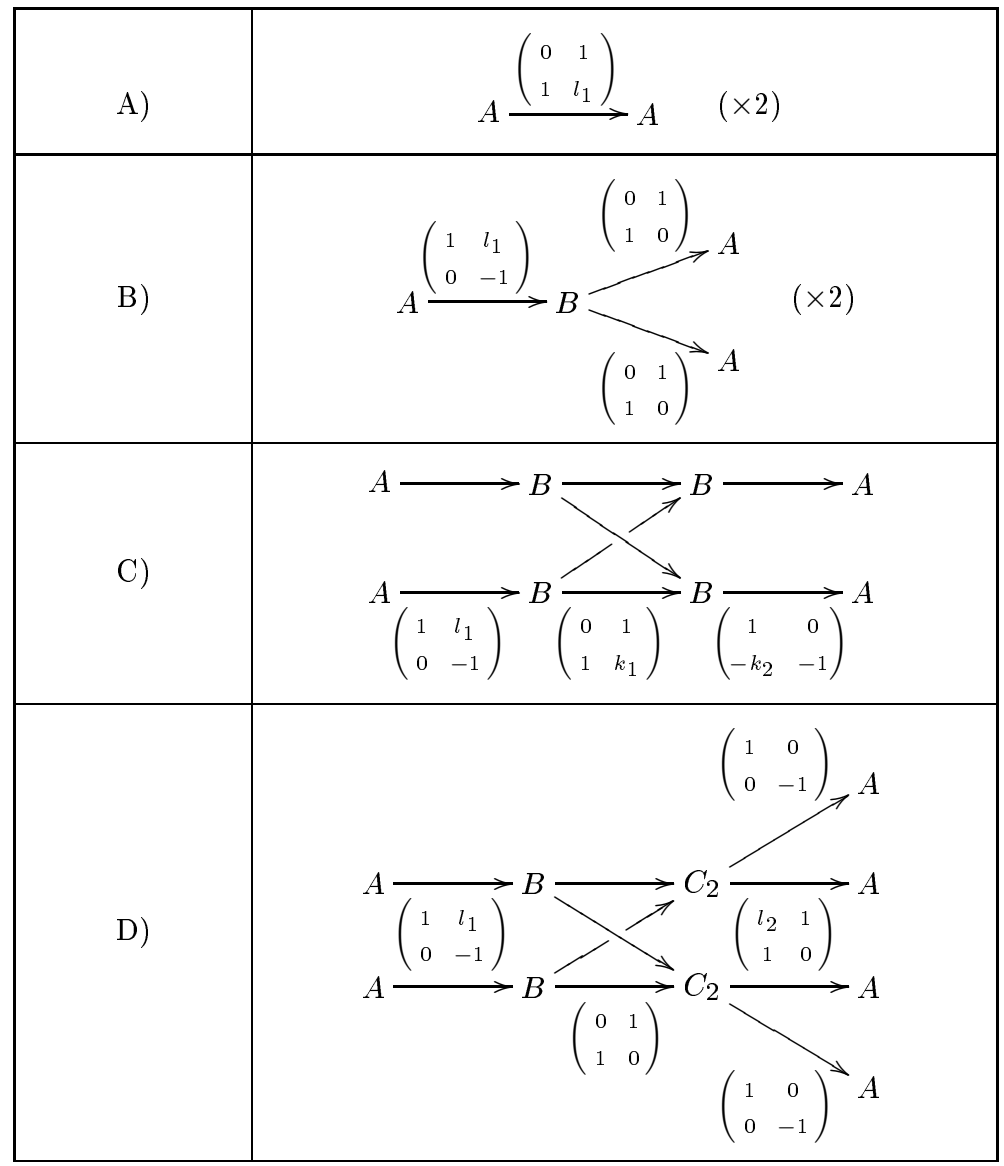


ТАБлицА 7 (продолжение)

\begin{tabular}{|c|c|}
\hline E) & $A \stackrel{\left(\begin{array}{ll}0 & 1 \\
1 & 0\end{array}\right)}{\longrightarrow} A$ \\
\hline $\mathrm{F})$ & $A \underset{\left(\begin{array}{ll}0 & 1 \\
1 & l_{1}\end{array}\right)}{\longrightarrow} C_{2}{ }_{\left(\begin{array}{cc}0 & -1 \\
-1 & k_{3}\end{array}\right)}^{A^{*}} A^{*} \stackrel{\left(\begin{array}{cc}-k_{4} & 1 \\
1 & 0\end{array}\right)}{\longrightarrow} A$ \\
\hline G) & $A \underset{\left(\begin{array}{ll}1 & l_{1} \\
0 & -1\end{array}\right)}{\longrightarrow} B \underset{\left(\begin{array}{ll}0 & 1 \\
1 & 0\end{array}\right)}{\longrightarrow} C_{2} \underset{\left(\begin{array}{cc}1 & 0 \\
0 & -1\end{array}\right)}{\longrightarrow} A$ \\
\hline $\mathrm{H})$ & A \\
\hline I) & $\underset{\left(\begin{array}{ll}0 & 1 \\
1 & l_{1}\end{array}\right)}{\longrightarrow} C_{2} \underset{\left(\begin{array}{cc}1 & 0 \\
0 & -1\end{array}\right)}{\longrightarrow} A$ \\
\hline
\end{tabular}


ТАБЛИЦА 8

\begin{tabular}{|c|c|c|c|}
\hline A) & $A \stackrel{\substack{r=0 \\
\varepsilon=1}}{\longrightarrow} A \quad(\times 2)$ & F) & 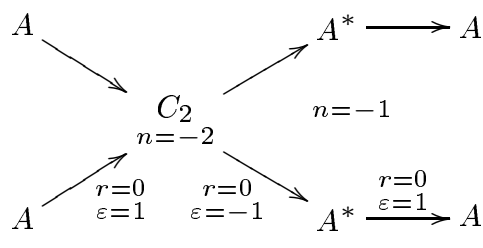 \\
\hline B) & 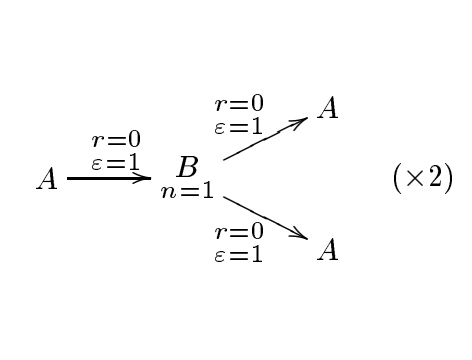 & G) & $A \longrightarrow \underset{\substack{r=0 \\
\varepsilon=1}}{\longrightarrow} B \underset{\substack{r=0 \\
\varepsilon=1}}{\longrightarrow} C_{\substack{r=\infty \\
\varepsilon=1}} C_{A} C_{A}$ \\
\hline C) & $A \underset{\substack{r=0 \\
\varepsilon=1}}{\longrightarrow} B \underset{\substack{r=0 \\
\varepsilon=1}}{\longrightarrow} B \underset{\substack{r=\infty \\
\varepsilon=1}}{\longrightarrow} A$ & $\mathrm{H})$ & $A \sum_{\substack{r=0 \\
\varepsilon=1}}^{\substack{C_{2} \\
\varepsilon=-2}} A$ \\
\hline D) & $A \underset{\substack{r=0 \\
\varepsilon=1}}{\longrightarrow} B \underset{\substack{r=0 \\
\varepsilon=1}}{\longrightarrow} C_{\substack{r=\infty \\
\varepsilon=1}} C_{A} C_{A}$ & I) & $\overbrace{\substack{r=0 \\
\varepsilon=1}}^{A} C_{\substack{r=\infty \\
\varepsilon=1}}^{A} A$ \\
\hline E) & $A \underset{\substack{C_{2}=0 \\
\varepsilon=1}}{\substack{r=0 \\
\varepsilon=-1}} A \underset{\substack{r=1 / 2 \\
\varepsilon=1}}{\longrightarrow} A$ & $\mathrm{~J})$ & $A \stackrel{\begin{array}{r}r=\infty \\
\varepsilon=-1\end{array}}{\longrightarrow} A$ \\
\hline K) & $A \underset{\substack{r=\infty \\
\varepsilon=1}}{\longrightarrow} \underset{\substack{r=\infty \\
\varepsilon=1}}{\longrightarrow} B \underset{\substack{r=\infty \\
\varepsilon=1}}{\longrightarrow} A$ & L) & $A \underset{\substack{r=0 \\
\varepsilon=1}}{\longrightarrow} A^{*} \underset{\substack{r=\infty \\
\varepsilon=1}}{\stackrel{n=-2}{\longrightarrow}} A^{*} \underset{\substack{r=0 \\
\varepsilon=1}}{\longrightarrow} A$ \\
\hline
\end{tabular}


4.8. Случай нулевой постоянной площадей. Для завершения описания инвариантов Фоменко-Цишанга системы Соколова осталось рассмотреть случай $g=0$. Его специфика заключается в том, что в прообразе левой стенки бифуркационной диаграммы лежат не критические боттовские окружности, а минимаксные торы (см. [10]). При подходе к этой стенке тор Лиувилля не испытывает бифуркации и после прохождения критического тора переходит в симметричный себе относительно инволюции $\Phi$. По этому семейству торов атомы $A_{\alpha_{2}}, B_{\beta_{1}}$ и $A_{\delta_{2}}^{*}$ склеиваются со вторыми своими симметричньми экземплярами. Следовательно, соответствующие метки $r=\infty$, а метки $\varepsilon=-1$ для атомов $A$ и $\varepsilon=+1$ для седловых атомов. В остальных семействах при переходе к нулевому $g$ характер перестроек не меняется и метки остаются прежними.

Три новые молекулы, появляюшиеся при $g=0$, обозначим J, K, L (см. рис. 7 и таблицу 8). Метки молекул $\mathrm{J}$ и $\mathrm{K}$ в силу изложенных выше соображений известны. Теперь можно определить топологические типы их поверхностей. Они таковы: $\mathrm{J}-2\left(\mathbb{S}^{1} \times \mathbb{S}^{2}\right), \mathrm{K}-\left(\mathbb{S}^{1} \times \mathbb{S}^{2}\right) \#\left(\mathbb{S}^{1} \times \mathbb{S}^{2}\right) \#\left(\mathbb{S}^{1} \times \mathbb{S}^{2}\right)$. Топологический тип L как молекулы максимальной энергии уравнений Кирхгофа известен: это $\mathbb{R} P^{3}$. В рассматриваемом случае метка $n$ семьи $A^{*}-A^{*}$ имеет смысл эйлерова класса многообразия $Q^{3}$. Значит, $n= \pm 2$ (в зависимости от выбора ориентации на $Q^{3}$ ). Остальные метки молекулы L очевидны.

Сформулируем итоговый результат этого параграфа.

Теорема 12. Полный список инвариантов Фоменко-Цишанга для случая Соколова приведен в табличе 8.

\section{Список литературы}

1. Болсинов A.B., Фоменко A.T. Интегрируемые гамильтоновы системы. Геометрия. Топология. Классификация. Т. 1, 2. Ижевск: Издательский дом "Удмуртский университет", 1999.

2. Стеклов B. A. О движении твердого тела в жидкости. Харьков, 1893.

3. Соколов В. В. Новый интегрируемый случай для уравнений Кирхгоффа // ТМФ. 2001. T. 129. № 1. C. 31-37.

4. Euler L. Du mouvement de rotation des corps solides autour d'un axe variable // Memoires de l'Academie des Sciences de Berlin. 1758. V. 14. P. 154-193.

5. Clebsch A. Über die Bewegung eines Körpers in einer Flüssigkeit // Math. Ann. Leipzig. 1871. V. 3. P. 238-262.

6. Морозов П. В. Лиувиллева классификация интегрируемых систем случая Клебша // Матем. сб. 2002. Т. 193. № 10. С. 113-138.

7. Болсинов А.В., Рихтер П., Фоменко А.Т. Метод круговых молекул и топология волчка Ковалевской // Матем. сб. 2000. Т. 191. № 2. С. 3-42.

8. Ошемков $A$. A. Описание изоэнергетических поверхностей интегрируемых гамильтоновых систем с двумя степенями свободы // Труды семинара по векторному и тензорному анализу. Т. 23. М.: МГУ, 1988. С. 122-132.

9. Ошемков A. A. Вычисление инварианта Фоменко для основных интегрируемых случаев динамики твердого тела // Труды семинара по векторному и тензорному анализу. Т. 25 . Часть 2. М.: МГУ, 1993. С. 23-110.

10. Рябов П. Е. Бифуркации первых интегралов в случае Соколова // ТМФ. 2003. Т. 134. № 2. C. 207-226.

11. Орел O. E. Функция вращения для интегрируемых задач, сводящихся к уравнениям Абеля. Траекторная классификация систем Горячева-Чаплыгина // Матем. сб. 1995. Т. 186. № 2. C. 105-128. 
12. Топалов П. Вычисление тонкого инварианта Фоменко-Цишанга для основных интегрируемых случаев движения твердого тела // Матем. сб. 1996. Т. 187. № 3. С. 143-160.

13. Nguyen Tien Zung. Decomposition of non-degenerate singularities of integrable Hamiltonian systems // Lett. Math. Phys. 1995. V. 33. P. 187-193.

14. Nguyen Tien Zung. A note on degenerate corank-one singularities of integrable Hamiltonian systems // Comment. Math. Helv. 2000. V. 75. P. 271-283.

Московский государственньй университет

им. М.В. Ломоносова

Поступила в редакцию

E-mail: morozov@rambler.ru

09.06 .2003 